\title{
Geochemical Characterization of the Paleocene Ewekoro Limestone Formation, SW Nigeria: Implications for Provenance, Diagenesis and Depositional Environment
}

\author{
O. Ajayi Ehinola', O. Innocent Ejeh ${ }^{2}$, O. Justine Oderinde ${ }^{1}$ \\ ${ }^{1}$ Department of Geology, University of Ibadan, Ibadan, Nigeria \\ ${ }^{2}$ Department of Geology, Delta State University, Abraka, Nigeria \\ Email: ehinola01@yahoo.com
}

Received 31 March 2016; accepted 17 July 2016; published 20 July 2016

Copyright (C) 2016 by authors and Scientific Research Publishing Inc.

This work is licensed under the Creative Commons Attribution International License (CC BY). http://creativecommons.org/licenses/by/4.0/

(c) (i) Open Access

\section{Abstract}

The usefulness of limestone as an industrial raw material is dependent on the level of its purity, largely controlled by diagenesis and the depositional setting. Limestone samples (83) obtained from the Ewekoro Formation exposed in quarry sections at Shagamu were analyzed using X-Ray Fluorescence spectrometry (XRF). A geochemical characterization of result was done to evaluate the purity levels and the implications on provenance, diagenesis, and depositional setting of the limestone. Five limestone beds from quarry sections (15 locations) were logged. Beds " $E$ ", “ $D$ ", “C”, " $B$ ", and " $A$ " comprised sandy algal in sparry calcite cement, sandy biomicrite, algal biomicrite, sandy algal biomicrite, and the intra-sparite facies respectively. Results of major elements chemistry indicated concentrations (in wt\%) as follows: $\mathrm{CaO}(33.71-59.99), \mathrm{MgO}(0.39-3.15), \mathrm{Al}_{2} \mathrm{O}_{3}$ $(0.47-3.23), \mathrm{Fe}_{2} \mathrm{O}_{3}(0.51-3.43), \mathrm{SiO}_{2}(0.47-45.98), \mathrm{SO}_{3}(0.10-2.27), \mathrm{K}_{2} \mathrm{O}(0.02-0.28), \mathrm{Na}_{2} \mathrm{O}(0.00-$ 10.0), $\mathrm{TiO}_{2}(0.00-0.27), \mathrm{P}_{2} \mathrm{O}_{5}(0.02-0.92), \mathrm{MnO}(0.01-0.06)$ and Loss on ignition (17.64 - 45.20). Geochemical result showed that the samples are enriched in $\mathrm{CaO}$, while $\mathrm{SiO}_{2}$ varies widely, likely due to hinterland input. Plots of $\mathrm{SiO}_{2}$ versus $\mathrm{CaO}$ concentrations depicted a negative correlation attributable to chemical diagenetic processes. Diagenesis of the carbonate in form of reversible replacement of $\mathrm{SiO}_{2}$ with $\mathrm{CaO}$ and vice versa occurred under a shallow marine condition. This negative correlation between these oxides $\left(\mathrm{CaO}\right.$ and $\left.\mathrm{SiO}_{2}\right)$ dictates zones of high purity limestones that are of desirable industrial applications. Relatively high silica content in the northwestern part is possible indication of outlets to the continental or inland areas where clastic sediment source may have been prominent. Ratios of $\mathrm{Ca} / \mathrm{Mg}$ and $\mathrm{Mg} / \mathrm{Ca}$ indicated a relatively low rate of evaporation of sea water and palaeo-salinity conditions marked by precipitation of limestone. Intermittent increase in palaeo-salinity and sea water evaporation level gave rise to the magnesian limestone. 
Keywords

Geochemical Characterization, Ewekoro Formation, Ca/Mg Ratio, Limestone, Carbonate, Silica

\section{Introduction}

Carbonate rocks constitute about 50\% of the world's hydrocarbon reservoir rocks, and among these limestones are widely used as raw materials in the chemical, metallurgical and construction industries. The quality and hence the usefulness of limestone deposit is largely dependent on the geological setting and the physico-chemical, mechanical and mineralogical characteristics of the stone. An evaluation of usefulness or appraisal of a limestone deposit entails a geological field investigation and laboratory analyses of representative samples. Naturally, limestone carries varied suite of impurities such as $\mathrm{SiO}_{2}, \mathrm{MgO}$ and $\mathrm{Fe}_{2} \mathrm{O}_{3}$, whose geochemical concentration determines its industrial application(s). Therefore an assessment of its grade through geochemical analyses such as $\mathrm{XRF}$ is essential. Most limestone industrial applications consider the carbonate and MgO contents as fundamental criteria for its chemical purity or grade classification.

In accord with the global increase in the applications of geological models for exploration and exploitation of mineral resources; geochemical models revealing limestone's chemical purity can be used as a tool to appraise the spatio-temporal distribution of limestone purity throughout the deposit. The modelling approach is targeted at locating anomalous concentration(s) of high purity limestone or other pathfinder elements and characterizing the host lithologies. This method may form a basis for comparison of data for limestones of other geological settings all over the earth.

The Ewekoro limestone belt extends to the northwest and beyond Shagamu to the southeastern part of the embayment. Nwajide [1] reported the limestone reserve estimate of [2] of about 36 million tons. Reyment [3] also presented estimated values of chemical constituents of quarried limestones from the Ewekoro as follows: $\mathrm{CaO}$ (53\%), $\mathrm{CO}_{2}$ (42\%), $\mathrm{SiO}_{2}$ (2\%), $\mathrm{Al}_{2} \mathrm{O}_{3}(5 \%), \mathrm{Fe}_{2} \mathrm{O}_{3}$ (1.4\%), $\mathrm{P}_{2} \mathrm{O}_{5}(0.8 \%), \mathrm{MgO}(0.3 \%), \mathrm{MnO}(0.1 \%)$, and minor quantities of $\mathrm{Na}_{2} \mathrm{O}, \mathrm{K}_{2} \mathrm{O}, \mathrm{TiO}_{2}, \mathrm{~F}$ and trace of $\mathrm{SO}_{3}$. Although, these geochemical reports are some five decades old and some of the relatively recent related studies include those of [4]-[7] and others; but not much has been done on geochemical characterization to re-appraise the spatio-temporal variation of elemental compositions of the carbonates. This study examines the geochemical characteristics of the Paleocene Ewekoro limestone Formation; using results from XRF analysis of samples obtained in order to determine provenance, diagenesis and depositional setting of the study area. The study area lies within Latitudes $6^{\circ} 47^{\prime} \mathrm{N}$ to $6^{\circ} 48^{\prime} \mathrm{N}$ and Longitudes $3^{\circ} 38^{\prime} \mathrm{E}$ to $3^{\circ} 39^{\prime} \mathrm{E}$, the present location of the Shagamu quarry (Figure 1).

\section{Geological Setting}

\subsection{Tectonic Framework}

The Dahomey Embayment spans the continental margin of the Gulf of Guinea, covering the Volta delta in Ghana to the west and the Okitipupa ridge/Benin hinge line to the east [8] [9]. It's a marginal pull-apart basin or marginal sag basin [10] that developed in the Mesozoic sequel to the separation of African from the South American plates [11] [12]. This separation, accompanied by basement fracturing accounted for the early rifting stage during Jurassic to Early Cretaceous and the development of several marginal sub-basins [9].

\subsection{Stratigraphy}

The eastern Dahomey Embayment (Figure 1) has been studied both on outcrop scale as well as from core holes by various workers such as [2] [4] [8] [9] [13]-[16], amongst others. The stratigraphy of the Nigerian sector of the embayment can be broadly divided into two: the Cretaceous Abeokuta Group (comprising Ise, Afowo and Araromi Formations) and the Cenozoic units (comprising Ewekoro, Akinbo, Oshoshun, Ilaro, and Benin Formations) [2] [9] (Figure 2). However, this study is focused on the Ewekoro limestone Formation.

This formation is made up of fossiliferous shelly limestone of about 12.5 meters thick, which tends to be sandy at the base [1]. It has been divided into three micro-facies namely: the sandy biomicrite lower unit; the shelly biomicrite grading into biomicrosparite middle unit that consists mainly of pure limestone making up the bulk of the 


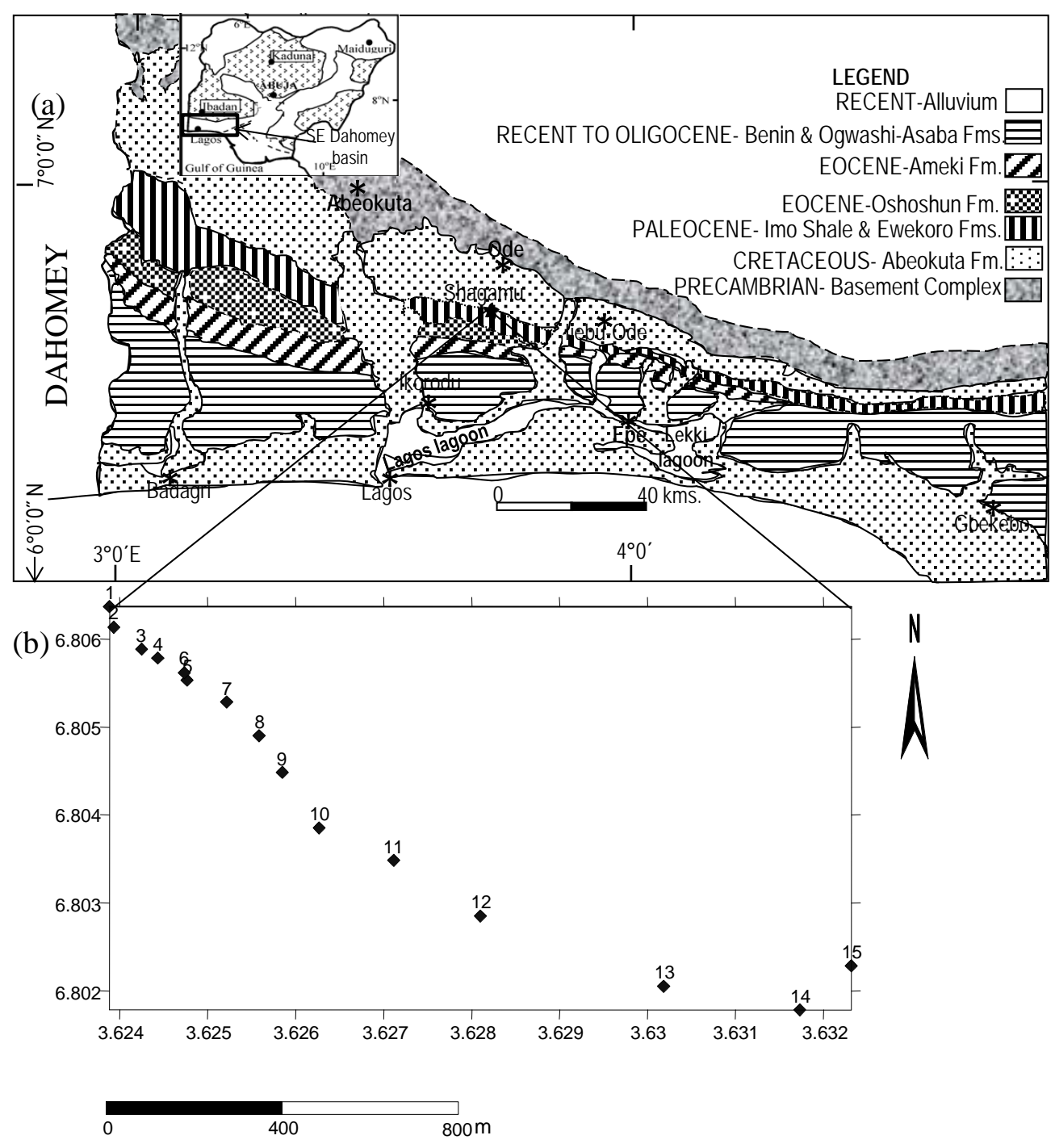

Figure 1. (a) Geological map of eastern Dahomey Embayment (redrawn after [11]; (b) Study area map with NW-SE trending sample locations.

Ewekoro Formation and; the shelly biomicrite and Algal biosparite upper unit [1]. It's of Paleocene age based on fossil evidence (foraminifera and ostracods) and deposited in a shallow marine environment [16] [17].

\section{Materials and Methods}

This work involved a geological field survey of the study area, a geochemical analysis and a computer-based geo-modelling to evaluate the $\mathrm{CaO}$ and $\mathrm{SiO}_{2}$ distribution in the limestone formation. The field work entailed examination and logging of quarry sections at Shagamu and 83 representative samples were obtained for laboratory analysis. Sampling was done from bottom to top at distinct limestone beds in fifteen sections in a NW-SE traverse (Figure 1(b)) and readings of geographical location obtained using a GPS device.

Sample preparation and analysis were done in the field-based laboratory of West African Portland Cement, Shagamu. Samples were washed, air dried, ground to powder form and homogenized; thereafter $2 \mathrm{~g}$ of each sample was mixed with spectroflux powder and $0.6 \mathrm{~g}$ of $\mathrm{LiNO}_{3}$ salt in an agate mortar. The mixture was poured into fusing containers on a burner within M4 fluxer equipment and switched on for fifteen minutes to produce fused pellets. Fused pellets produced were analyzed for major elements using an X-Ray fluorescence machine (ARL $9900 \mathrm{XP}$ ). Loss on ignition (LOI) was determined separately by calculating weight loss after heating $2 \mathrm{~g}$ of each sample in a furnace for 1 hour at $1000^{\circ} \mathrm{C}$. 


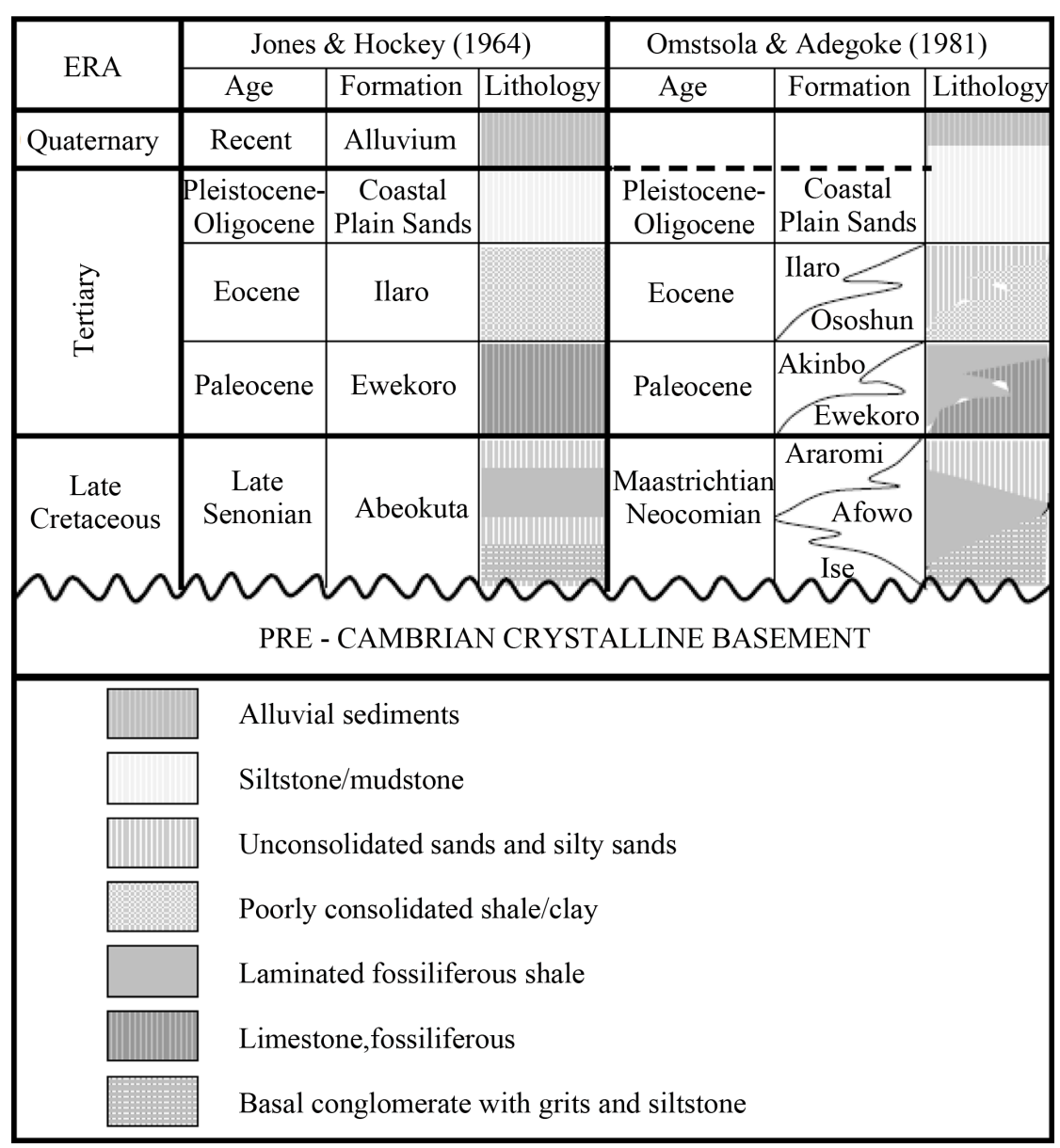

Figure 2. Stratigraphy of the Dahomey Embayment (After [2] and [9]).

The geochemical results obtained (Table 1) were further evaluated using a computer programme (Surfer ${ }^{T M}$ ) to generate geochemical models for the distribution of $\mathrm{CaO}$ and $\mathrm{SiO}_{2}$ (the main component and impurity in limestones respectively). To achieve this, the latitudinal and longitudinal readings were scaled to the $\mathrm{X}$ and $\mathrm{Y}$-axes respectively, while the corresponding chemical concentrations (in wt\%) of $\mathrm{CaO}$ and $\mathrm{SiO}_{2}$ were scaled to the Z-axis for each of the beds. Through a statistical algorithm called kriging, the GPS readings and concentrations of $\mathrm{CaO}$ and $\mathrm{SiO}_{2}$ were interpolated, resulting in a spatio-temporal distribution of these oxides within the various beds through a grid-based contouring of their concentrations. This variogram mathematically express the variance of the geochemical concentration in each of the beds giving a series of surface geochemical models that may constitute baseline information for further exploitation of the deposit.

\section{Results and Interpretations}

\subsection{Field Relationships}

Field study revealed five limestone beds, labeled E to A spanning the 15 sections logged (Figure 1 and Figure 3); although a sixth bed, F (which is quite silty with large amount of quartz) was recognized belonging to the underlying Araromi Formation. Bed E is the oldest bed among the limestone units. It's a light brown sandy algal bed with fossils embedded in sparry calcite cement. Bed D is light brown, shelly biomicritic and inter-fingered Bed $\mathrm{E}$ which is more friable, sandy algal biosparite, with the cementing material mostly sparry calcite. Bed C is dark grey, calcareous unit that comprised algal biomicrite facies. Bed B is grayish to brown in colour, hard with greater tenacity than the overlying bed and comprised sandy algal biomicrite embedded in micrite cement. Bed A is a red phosphatic limestone (typifying the intra-sparite facies) that is crystalline textured with localized quartz fillings within vugs and caves induced by migrating acidic fluids. 
Table 1. Major elements composition (wt\%) of Limestones from the Ewekoro Formation exposed at Shagamu.

\begin{tabular}{|c|c|c|c|c|c|c|c|c|c|c|c|c|c|c|}
\hline $\begin{array}{c}\text { Bed } \\
\text { Name }\end{array}$ & $\begin{array}{l}\text { LOI } \\
(\%)\end{array}$ & $\begin{array}{l}\mathrm{SiO}_{2} \\
(\%)\end{array}$ & $\begin{array}{c}\mathrm{Al}_{2} \mathbf{O}_{3} \\
(\%)\end{array}$ & $\begin{array}{c}\mathrm{Fe}_{2} \mathbf{O}_{3} \\
(\%)\end{array}$ & $\begin{array}{c}\mathrm{CaO} \\
(\%)\end{array}$ & $\begin{array}{c}\text { MgO } \\
(\%)\end{array}$ & $\begin{array}{l}\mathrm{SO}_{3} \\
(\%)\end{array}$ & $\begin{array}{l}\mathrm{K}_{2} \mathrm{O} \\
(\%)\end{array}$ & $\begin{array}{c}\mathrm{Na}_{2} \mathrm{O} \\
(\%)\end{array}$ & $\begin{array}{l}\mathrm{TiO}_{2} \\
(\%)\end{array}$ & $\begin{array}{c}\mathbf{P}_{2} \mathbf{O}_{5} \\
(\%)\end{array}$ & $\begin{array}{c}\mathrm{MnO} \\
(\%)\end{array}$ & $\begin{array}{c}\mathrm{Cr}_{2} \mathrm{O}_{3} \\
(\%)\end{array}$ & $\begin{array}{c}\text { Total } \\
(\%)\end{array}$ \\
\hline \multicolumn{15}{|c|}{ Location 1} \\
\hline Bed A1 & 44.89 & 00.88 & 0.98 & 0.61 & 52.01 & 0.62 & 0.18 & 0.04 & 00.00 & 0.01 & 0.08 & 0.04 & 0.00 & 100.3 \\
\hline Bed B1 & 44.52 & 01.98 & 1.59 & 1.28 & 48.78 & 0.83 & 0.92 & 0.06 & 00.00 & 0.03 & 0.30 & 0.02 & 0.00 & 100.3 \\
\hline Bed C1 & 44.52 & 01.98 & 1.59 & 1.28 & 48.78 & 0.81 & 0.47 & 0.06 & 00.00 & 0.08 & 0.12 & 0.01 & 0.00 & 100.7 \\
\hline Bed D1 & 41.67 & 01.85 & 0.52 & 0.62 & 54.22 & 0.64 & 0.15 & 0.06 & 00.20 & 0.03 & 0.04 & 0.01 & 0.00 & 100.0 \\
\hline Bed E1 & 40.38 & 05.22 & 0.55 & 1.42 & 49.98 & 2.26 & 0.15 & 0.06 & 00.00 & 0.04 & 0.06 & 0.04 & 0.00 & 100.2 \\
\hline Bed F1 & 42.17 & 09.15 & 2.44 & 1.33 & 42.84 & 1.40 & 0.64 & 0.08 & 00.00 & 0.10 & 0.17 & 0.02 & 0.00 & 100.4 \\
\hline \multicolumn{15}{|c|}{ Location 2} \\
\hline Bed A2 & 42.05 & 01.40 & 1.07 & 0.86 & 53.97 & 0.72 & 0.29 & 0.03 & 00.00 & 0.01 & 0.08 & 0.04 & 0.00 & 100.5 \\
\hline Bed B2 & 43.69 & 01.10 & 0.95 & 0.54 & 52.85 & 0.78 & 0.24 & 0.05 & 00.00 & 0.02 & 0.09 & 0.01 & 0.00 & 100.3 \\
\hline Bed C2 & 37.70 & 10.65 & 1.63 & 1.72 & 46.73 & 0.87 & 0.92 & 0.13 & 00.00 & 0.15 & 0.25 & 0.01 & 0.00 & 100.8 \\
\hline Bed D2 & 40.25 & 05.34 & 0.55 & 0.64 & 52.70 & 0.64 & 0.10 & 0.06 & 00.20 & 0.02 & 0.04 & 0.01 & 0.00 & 100.6 \\
\hline Bed E2 & 40.25 & 06.34 & 1.96 & 1.82 & 46.33 & 3.15 & 0.42 & 0.08 & 00.00 & 0.13 & 0.11 & 0.04 & 0.00 & 100.6 \\
\hline \multicolumn{15}{|c|}{ Location 3} \\
\hline Bed A3 & 41.56 & 01.51 & 0.90 & 0.67 & 54.24 & 1.24 & 0.39 & 0.02 & 00.00 & 0.01 & 0.08 & 0.04 & 0.00 & 100.7 \\
\hline Bed B3 & 43.90 & 01.57 & 0.63 & 1.01 & 50.05 & 3.31 & 0.17 & 0.05 & 00.00 & 0.03 & 0.03 & 0.01 & 0.00 & 100.8 \\
\hline Bed C3 & 29.93 & 22.15 & 3.04 & 3.06 & 38.81 & 3.14 & 0.74 & 0.25 & 00.00 & 0.27 & 0.27 & 0.01 & 0.00 & 100.7 \\
\hline Bed D3 & 31.14 & 31.21 & 1.64 & 1.05 & 34.22 & 0.62 & 0.23 & 0.07 & 00.00 & 0.06 & 0.24 & 0.03 & 0.00 & 100.5 \\
\hline Bed E3 & 40.25 & 06.34 & 1.96 & 1.82 & 46.33 & 3.15 & 0.42 & 0.08 & 00.00 & 0.13 & 0.11 & 0.04 & 0.00 & 100.6 \\
\hline \multicolumn{15}{|c|}{ Location 4} \\
\hline Bed A4 & 43.29 & 01.15 & 0.53 & 0.67 & 52.75 & 1.72 & 0.30 & 0.03 & 00.00 & 0.01 & 0.09 & 0.01 & 0.00 & 100.6 \\
\hline Bed D4 & 31.79 & 27.58 & 1.57 & 1.24 & 37.05 & 0.74 & 0.21 & 0.07 & 10.00 & 0.11 & 0.20 & 0.03 & 0.00 & 100.6 \\
\hline Bed E4 & 43.83 & 02.58 & 1.23 & 0.83 & 50.48 & 0.75 & 0.78 & 0.04 & 10.00 & 0.04 & 0.10 & 0.01 & 0.00 & 100.7 \\
\hline \multicolumn{15}{|c|}{ Location 5} \\
\hline Bed A5 & 43.29 & 01.15 & 0.53 & 0.67 & 52.70 & 1.72 & 0.30 & 0.03 & 00.00 & 0.01 & 0.09 & 0.02 & 0.00 & 100.2 \\
\hline Bed B5 & 43.93 & 01.57 & 0.63 & 1.01 & 50.04 & 3.31 & 0.17 & 0.05 & 00.00 & 0.03 & 0.02 & 0.01 & 0.00 & 100.8 \\
\hline Bed C5 & 31.14 & 22.23 & 3.07 & 3.43 & 36.84 & 2.05 & 0.82 & 0.28 & 00.00 & 0.20 & 0.34 & 0.01 & 0.00 & 100.4 \\
\hline Bed D5 & 32.79 & 26.58 & 1.57 & 1.24 & 37.06 & 0.74 & 0.21 & 0.07 & 00.00 & 0.11 & 0.24 & 0.01 & 0.00 & 100.6 \\
\hline Bed E5 & 43.81 & 02.58 & 1.23 & 0.83 & 50.49 & 0.75 & 0.78 & 0.04 & 00.00 & 0.04 & 0.10 & 0.01 & 0.00 & 100.6 \\
\hline \multicolumn{15}{|c|}{ Location 6} \\
\hline Bed A6 & 40.92 & 02.07 & 1.73 & 0.68 & 52.58 & 1.03 & 0.77 & 0.06 & 00.00 & 0.00 & 0.22 & 0.05 & 0.00 & 100.1 \\
\hline Bed B6 & 45.10 & 01.26 & 1.64 & 0.51 & 50.01 & 1.01 & 0.31 & 0.05 & 00.00 & 0.02 & 0.10 & 0.01 & 0.00 & 100.0 \\
\hline Bed C6 & 31.14 & 22.23 & 3.07 & 3.43 & 35.84 & 2.05 & 0.82 & 0.28 & 00.00 & 0.20 & 0.34 & 0.02 & 0.00 & 99.42 \\
\hline
\end{tabular}




\section{Continued}

\begin{tabular}{|c|c|c|c|c|c|c|c|c|c|c|c|c|c|c|}
\hline $\begin{array}{c}\text { Sample } \\
\text { code }\end{array}$ & $\begin{array}{l}\text { LOI } \\
(\%)\end{array}$ & $\begin{array}{l}\mathrm{SiO}_{2} \\
(\%)\end{array}$ & $\begin{array}{c}\mathrm{Al}_{2} \mathrm{O}_{3} \\
(\%)\end{array}$ & $\begin{array}{c}\mathrm{Fe}_{2} \mathrm{O}_{3} \\
(\%)\end{array}$ & $\begin{array}{c}\mathrm{CaO} \\
(\%)\end{array}$ & $\begin{array}{c}\text { MgO } \\
\text { (\%) }\end{array}$ & $\begin{array}{l}\mathrm{SO}_{3} \\
(\%)\end{array}$ & $\begin{array}{l}\mathrm{K}_{2} \mathrm{O} \\
(\%)\end{array}$ & $\begin{array}{c}\mathrm{Na}_{2} \mathrm{O} \\
(\%)\end{array}$ & $\begin{array}{l}\mathrm{TiO}_{2} \\
(\%)\end{array}$ & $\begin{array}{l}\mathbf{P}_{2} \mathbf{O}_{5} \\
(\%)\end{array}$ & $\begin{array}{c}\text { MnO } \\
(\%)\end{array}$ & $\begin{array}{c}\mathrm{Cr}_{2} \mathrm{O}_{3} \\
(\%)\end{array}$ & $\begin{array}{l}\text { Total } \\
\text { (\%) }\end{array}$ \\
\hline Bed D6 & 33.79 & 25.58 & 1.57 & 1.24 & 37.08 & 0.74 & 0.21 & 0.07 & 00.00 & 0.11 & 0.24 & 0.02 & 0.00 & 100.7 \\
\hline Bed E6 & 43.83 & 02.58 & 1.23 & 0.83 & 50.48 & 0.75 & 0.78 & 0.04 & 00.00 & 0.04 & 0.10 & 0.01 & 0.00 & 100.6 \\
\hline \multicolumn{15}{|c|}{ Location 7} \\
\hline Bed A7 & 40.92 & 02.07 & 1.73 & 0.68 & 52.57 & 1.03 & 0.77 & 0.06 & 00.00 & 0.00 & 0.22 & 0.02 & 0.00 & 100.1 \\
\hline Bed B7 & 45.20 & 01.26 & 1.64 & 0.51 & 50.20 & 1.01 & 0.31 & 0.05 & 00.00 & 0.02 & 0.10 & 0.01 & 0.00 & 100.3 \\
\hline Bed C7 & 32.14 & 21.23 & 3.07 & 3.43 & 36.84 & 2.05 & 0.82 & 0.28 & 00.00 & 0.20 & 0.34 & 0.02 & 0.00 & 100.1 \\
\hline Bed D7 & 31.79 & 27.58 & 1.57 & 1.24 & 37.09 & 0.74 & 0.21 & 0.07 & 00.00 & 0.11 & 0.24 & 0.01 & 0.00 & 100.7 \\
\hline Bed E7 & 43.83 & 02.55 & 1.23 & 0.83 & 50.45 & 0.75 & 0.78 & 0.04 & 00.00 & 0.04 & 0.10 & 0.01 & 0.00 & 100.6 \\
\hline Bed F7 & 42.17 & 09.15 & 2.44 & 1.33 & 42.84 & 1.40 & 0.64 & 0.08 & 00.00 & 0.10 & 0.17 & 0.01 & 0.00 & 100.3 \\
\hline \multicolumn{15}{|c|}{ Location 8} \\
\hline Bed A8 & 42.09 & 01.30 & 0.47 & 0.55 & 55.08 & 0.93 & 0.16 & 0.03 & 00.00 & 0.02 & 0.10 & 0.01 & 0.00 & 100.7 \\
\hline Bed B8 & 41.30 & 01.40 & 0.81 & 0.83 & 54.13 & 0.61 & 0.44 & 0.07 & 00.34 & 0.02 & 0.04 & 0.01 & 0.00 & 100.0 \\
\hline Bed C8 & 42.17 & 09.15 & 2.44 & 1.33 & 42.84 & 1.40 & 0.64 & 0.08 & 00.00 & 0.10 & 0.17 & 0.01 & 0.00 & 100.3 \\
\hline Bed D8 & 30.79 & 27.58 & 1.57 & 1.24 & 37.12 & 0.74 & 0.21 & 0.07 & 00.00 & 0.11 & 0.24 & 0.02 & 0.00 & 99.69 \\
\hline Bed E8 & 43.83 & 02.54 & 1.23 & 0.83 & 50.46 & 0.75 & 0.78 & 0.04 & 00.00 & 0.04 & 0.10 & 0.01 & 0.00 & 100.6 \\
\hline Bed F8 & 42.17 & 09.15 & 2.44 & 1.33 & 42.84 & 1.40 & 0.64 & 0.08 & 00.00 & 0.10 & 0.17 & 0.01 & 0.00 & 100.3 \\
\hline \multicolumn{15}{|c|}{ Location 9} \\
\hline Bed A9 & 43.19 & 00.47 & 0.37 & 0.88 & 54.20 & 0.39 & 0.21 & 0.02 & 0.00 & 0.01 & 0.92 & 0.05 & 0.00 & 100.7 \\
\hline Bed B9 & 33.58 & 09.98 & 1.53 & 3.34 & 48.19 & 0.73 & 2.27 & 0.17 & 0.00 & 0.15 & 0.70 & 0.01 & 0.00 & 100.7 \\
\hline Bed C9 & 36.41 & 09.91 & 1.84 & 1.72 & 49.17 & 0.88 & 1.24 & 0.11 & 0.00 & 0.11 & 0.28 & 0.01 & 0.00 & 100.7 \\
\hline Bed D9 & 31.80 & 27.58 & 1.57 & 1.24 & 37.05 & 0.74 & 0.21 & 0.07 & 0.00 & 0.11 & 0.24 & 0.01 & 0.00 & 100.6 \\
\hline Bed E9 & 43.83 & 02.59 & 1.23 & 0.83 & 50.49 & 0.75 & 0.78 & 0.04 & 0.00 & 0.04 & 0.10 & 0.01 & 0.00 & 100.7 \\
\hline Bed F9 & 42.17 & 09.15 & 2.44 & 1.33 & 42.84 & 1.40 & 0.64 & 0.08 & 0.00 & 0.10 & 0.17 & 0.01 & 0.00 & 100.3 \\
\hline \multicolumn{15}{|c|}{ Location 10} \\
\hline Bed A10 & 41.68 & 01.24 & 0.32 & 0.67 & 55.77 & 0.79 & 0.13 & 0.02 & 0.00 & 0.02 & 0.03 & 0.03 & 0.00 & 100.7 \\
\hline Bed B10 & 36.63 & 01.56 & 0.56 & 0.92 & 59.99 & 0.68 & 0.30 & 0.05 & 0.00 & 0.03 & 0.05 & 0.01 & 0.00 & 100.8 \\
\hline Bed C10 & 36.51 & 09.91 & 1.84 & 1.72 & 48.17 & 0.88 & 1.24 & 0.11 & 0.00 & 0.11 & 0.28 & 0.04 & 0.00 & 100.7 \\
\hline Bed D10 & 43.42 & 01.69 & 0.78 & 0.96 & 52.70 & 0.52 & 0.15 & 0.05 & 0.00 & 0.03 & 0.06 & 0.02 & 0.00 & 100.4 \\
\hline Bed E10 & 43.83 & 02.58 & 1.23 & 0.83 & 50.48 & 0.75 & 0.78 & 0.04 & 0.00 & 0.04 & 0.10 & 0.01 & 0.00 & 100.7 \\
\hline Bed F10 & 17.64 & 45.18 & 1.22 & 1.62 & 33.71 & 0.11 & 0.16 & 0.12 & 0.00 & 0.09 & 0.22 & 0.02 & 0.00 & 100.1 \\
\hline \multicolumn{15}{|c|}{ Location 11} \\
\hline Bed A11 & 41.68 & 01.24 & 0.32 & 0.67 & 55.75 & 0.79 & 0.13 & 0.02 & 0.00 & 0.02 & 0.03 & 0.01 & 0.00 & 100.7 \\
\hline Bed B11 & 38.47 & 02.94 & 0.94 & 0.94 & 55.08 & 0.62 & 0.69 & 0.07 & 0.16 & 0.06 & 0.04 & 0.01 & 0.00 & 100.0 \\
\hline
\end{tabular}




\section{Continued}

\begin{tabular}{|c|c|c|c|c|c|c|c|c|c|c|c|c|c|c|}
\hline Bed C11 & 42.53 & 07.58 & 1.63 & 1.36 & 45.29 & 0.87 & 1.00 & 0.09 & 0.00 & 0.10 & 0.25 & 0.01 & 0.00 & 100.7 \\
\hline Bed D11 & 36.41 & 09.91 & 1.84 & 1.72 & 48.19 & 0.88 & 1.24 & 0.11 & 0.00 & 0.11 & 0.28 & 0.01 & 0.00 & 100.7 \\
\hline Bed E11 & 37.32 & 01.68 & 0.93 & 0.67 & 53.30 & 0.72 & 0.20 & 0.05 & 0.00 & 0.02 & 0.09 & 0.03 & 0.00 & 100.1 \\
\hline Bed F11 & 17.64 & 45.18 & 1.22 & 1.62 & 33.71 & 0.11 & 0.16 & 0.12 & 0.00 & 0.09 & 0.22 & 0.02 & 0.00 & 100.1 \\
\hline \multicolumn{15}{|c|}{ Location 12} \\
\hline Bed A12 & 41.68 & 01.24 & 0.32 & 0.67 & 55.76 & 0.79 & 0.13 & 0.02 & 0.00 & 0.02 & 0.03 & 0.01 & 0.00 & 100.7 \\
\hline Bed B12 & 41.66 & 01.85 & 0.72 & 0.58 & 54.65 & 0.64 & 0.30 & 0.05 & 0.00 & 0.03 & 0.15 & 0.00 & 0.00 & 100.6 \\
\hline Bed C12 & 24.31 & 16.02 & 4.04 & 2.64 & 50.78 & 1.14 & 0.77 & 0.14 & 0.00 & 0.23 & 0.22 & 0.03 & 0.00 & 100.3 \\
\hline Bed D12 & 35.41 & 09.91 & 1.84 & 1.72 & 48.20 & 0.88 & 1.24 & 0.11 & 0.00 & 0.11 & 0.28 & 0.02 & 0.00 & 99.72 \\
\hline Bed E12 & 37.32 & 01.69 & 0.93 & 0.67 & 53.37 & 0.72 & 0.20 & 0.05 & 0.00 & 0.02 & 0.09 & 0.03 & 0.00 & 100.0 \\
\hline Bed F12 & 17.64 & 45.18 & 1.22 & 1.62 & 33.71 & 0.11 & 0.16 & 0.12 & 0.00 & 0.09 & 0.22 & 0.03 & 0.00 & 100.1 \\
\hline \multicolumn{15}{|c|}{ Location 13} \\
\hline Bed A13 & 43.99 & 00.79 & 0.76 & 0.63 & 53.54 & 0.50 & 0.16 & 0.02 & 0.00 & 0.01 & 0.10 & 0.05 & 0.00 & 100.6 \\
\hline Bed B13 & 42.74 & 01.27 & 0.45 & 0.75 & 54.42 & 0.63 & 0.21 & 0.04 & 0.00 & 0.02 & 0.07 & 0.00 & 0.00 & 100.6 \\
\hline Bed C13 & 37.09 & 03.51 & 1.00 & 1.05 & 56.52 & 1.02 & 0.41 & 0.04 & 0.00 & 0.06 & 0.06 & 0.00 & 0.00 & 100.8 \\
\hline Bed D13 & 36.41 & 09.91 & 1.84 & 1.72 & 48.18 & 0.88 & 1.24 & 0.11 & 0.00 & 0.11 & 0.28 & 0.00 & 0.00 & 100.7 \\
\hline Bed E13 & 42.59 & 01.69 & 0.93 & 0.67 & 53.39 & 0.72 & 0.20 & 0.05 & 0.00 & 0.02 & 0.09 & 0.01 & 0.00 & 100.4 \\
\hline Bed F13 & 17.64 & 45.18 & 1.22 & 1.62 & 33.71 & 0.11 & 0.16 & 0.12 & 0.00 & 0.09 & 0.22 & 0.02 & 0.00 & 100.1 \\
\hline \multicolumn{15}{|c|}{ Location 14} \\
\hline Bed A14 & 42.82 & 01.70 & 1.24 & 0.75 & 52.72 & 0.65 & 0.28 & 0.03 & 0.00 & 0.01 & 0.21 & 0.06 & 0.00 & 100.4 \\
\hline Bed B14 & 41.50 & 02.27 & 0.79 & 0.70 & 53.77 & 0.89 & 0.54 & 0.06 & 0.00 & 0.03 & 0.17 & 0.00 & 0.00 & 100.7 \\
\hline Bed C14 & 32.06 & 21.92 & 2.79 & 3.09 & 38.61 & 0.79 & 1.27 & 0.28 & 0.00 & 0.26 & 0.50 & 0.01 & 0.00 & 100.6 \\
\hline Bed D14 & 35.41 & 09.91 & 1.84 & 1.72 & 48.37 & 0.88 & 1.24 & 0.11 & 0.00 & 0.11 & 0.28 & 0.02 & 0.00 & 99.89 \\
\hline Bed E14 & 43.58 & 01.69 & 0.93 & 0.67 & 53.34 & 0.72 & 0.20 & 0.05 & 0.00 & 0.02 & 0.09 & 0.01 & 0.00 & 100.3 \\
\hline Bed F14 & 17.64 & 45.18 & 1.22 & 1.62 & 33.71 & 0.11 & 0.16 & 0.12 & 0.00 & 0.09 & 0.22 & 0.01 & 0.00 & 100.1 \\
\hline \multicolumn{15}{|c|}{ Location 15} \\
\hline Bed A15 & 42.82 & 01.70 & 1.24 & 0.75 & 52.71 & 0.65 & 0.28 & 0.03 & 0.00 & 0.01 & 0.21 & 0.06 & 0.00 & 100.4 \\
\hline Bed B15 & 43.90 & 01.12 & 0.72 & 1.52 & 50.74 & 2.33 & 0.16 & 0.04 & 0.00 & 0.02 & 0.03 & 0.02 & 0.00 & 100.6 \\
\hline Bed C15 & 33.06 & 21.92 & 2.79 & 3.09 & 36.61 & 0.79 & 1.27 & 0.28 & 0.00 & 0.26 & 0.50 & 0.01 & 0.00 & 100.6 \\
\hline Bed D15 & 36.31 & 09.91 & 1.84 & 1.72 & 48.17 & 0.88 & 1.24 & 0.11 & 0.00 & 0.11 & 0.28 & 0.01 & 0.00 & 100.6 \\
\hline Bed E15 & 42.58 & 01.69 & 0.93 & 0.67 & 53.38 & 0.72 & 0.20 & 0.05 & 0.00 & 0.02 & 0.09 & 0.01 & 0.00 & 100.4 \\
\hline Bed F15 & 17.64 & 45.18 & 1.22 & 1.62 & 33.71 & 0.11 & 0.16 & 0.12 & 0.00 & 0.09 & 0.22 & 0.02 & 0.00 & 100.1 \\
\hline
\end{tabular}

\subsection{Major Elements Distribution}

The concentrations (in wt $\%$ of oxides) of major elements in the limestone samples are shown in Table 1. The 


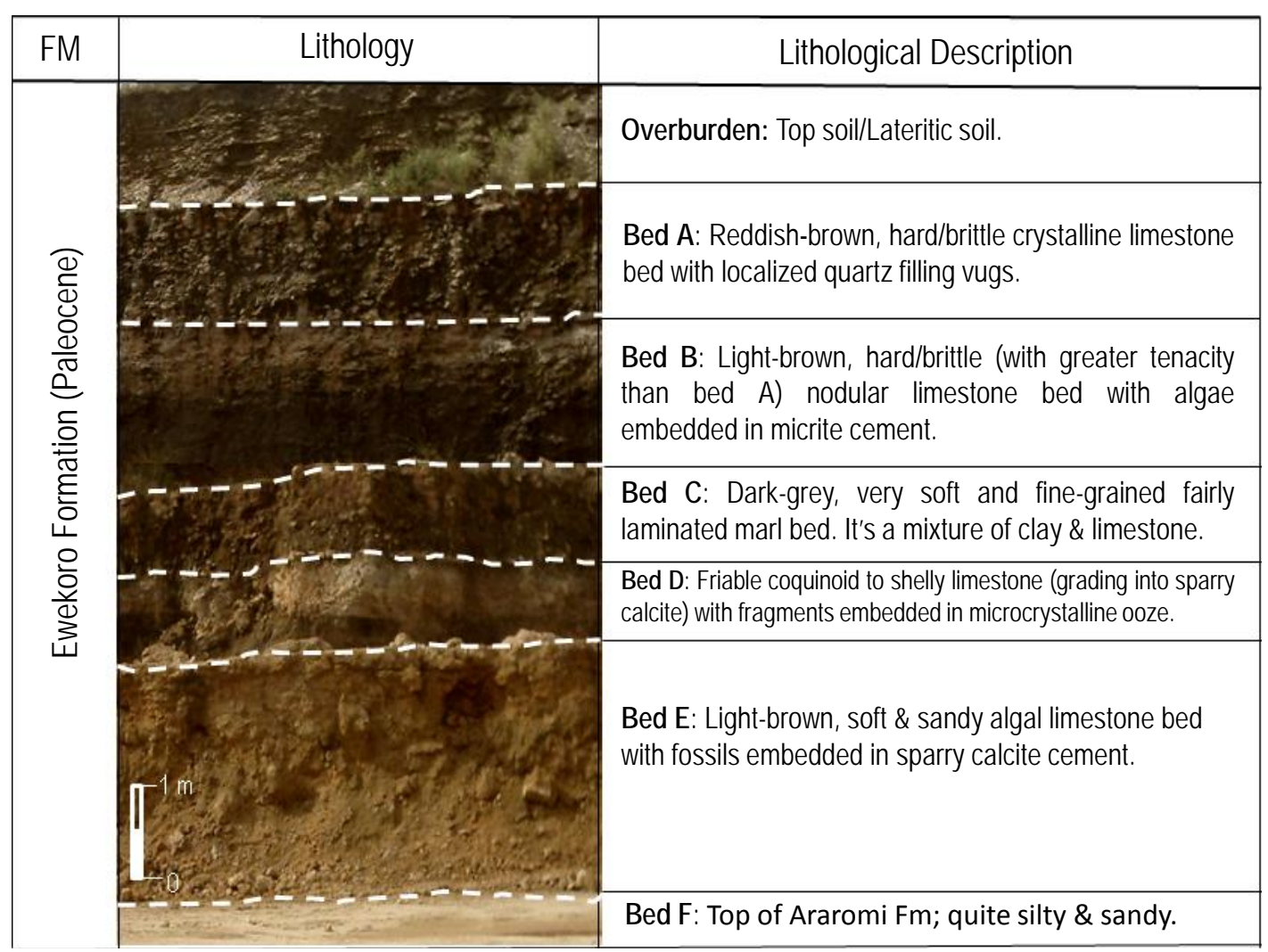

Figure 3. A representative lithologic section of the Ewekoro Formation (Fm) observed at Shagamu Quarry.

limestone samples analyzed are enriched (>1 wt\%) in CaO (33.7 - 59.99 wt\%, average 47.29 wt\%) and slightly depleted to enriched in $\mathrm{SiO}_{2}(0.47$ - $31.21 \mathrm{wt} \%), \mathrm{Al}_{2} \mathrm{O}_{3}$ (0.47 - $\left.3.23 \mathrm{wt} \%\right)$, $\mathrm{MgO}(0.39-3.15 \mathrm{wt} \%), \mathrm{Fe}_{2} \mathrm{O}_{3}(0.51-$ $3.43 \mathrm{wt} \%)$ and $\mathrm{SO}_{3}(0.10-2.27 \mathrm{wt} \%)$. However, they are depleted ( $\left.<1 \mathrm{wt} \%\right)$ in the alkalis $\left(\mathrm{Na}_{2} \mathrm{O}\right.$ and $\left.\mathrm{K}_{2} \mathrm{O}\right)$, $\mathrm{TiO}_{2}, \mathrm{MnO}, \mathrm{P}_{2} \mathrm{O}_{5}$ and $\mathrm{Cr}_{2} \mathrm{O}_{3}$. LOI varies between $17.64-45.10$ wt percent. The values of $\mathrm{CaO}$ and $\mathrm{MgO}$ tend to decrease down the Formation with increasing silica content probably due to the presence of non-carbonate input transported from adjacent continental sources.

\section{3. $\mathrm{CaO}$ and $\mathrm{SiO}_{2}$ Variations in the Ewekoro Limestone Formation}

A marked inverse negative correlation exists between $\mathrm{SiO}_{2}$ and $\mathrm{CaO}$ contents of the limestones (Figure 4). A generalized geochemical variation model for the area representing the $\mathrm{SiO}_{2}$ distribution is depicted in Figure 5 . A bed-by-bed spatio-temporal geochemical distribution of the concentrations of $\mathrm{CaO}$ and $\mathrm{SiO}_{2}$ is discussed below in super-positional order.

\subsection{1. $\mathrm{CaO}$ and $\mathrm{SiO}_{2}$ Variation in Bed $\mathrm{E}$}

Bed E is 1 - 2 m thick, occurring at depths of 17 - $38 \mathrm{~m}$ and 18 - $40 \mathrm{~m}$ at the upper and lower surfaces respectively. Table 1 shows that $\mathrm{CaO}$ content ranged from 46.33 - 53.39 wt\%, while $\mathrm{SiO}_{2}$ ranged from 1.68 to 6.34 wt\%. Figure 6 is the 2-D and 3-D model views of $\mathrm{CaO}$ and $\mathrm{SiO}_{2}$. A reduction in $\mathrm{CaO}$ concentration was noticed mostly in the south-central and northwestern part of the study area. However, an average abundance of CaO (>50 wt\%) was maintained in other portions of the bed keeping $\mathrm{SiO}_{2}$ proportions at minima level.

This zone carries the highest concentration and purest form of calcite. These calcite-rich zones could serve as suitable targets for limestone mining works, development and the production of Portland cement.

\subsection{2. $\mathrm{CaO}$ and $\mathrm{SiO}_{2}$ Variation in Bed D}

Bed $\mathrm{D}$ is $\approx 3-4 \mathrm{~m}$ thick and occurred at depths of 14 - $34 \mathrm{~m}$ and 17 - $38 \mathrm{~m}$ at the upper and lower surfaces respectively. From Table 1, the $\mathrm{CaO}$ contents ranged from 34.22 - 54.22 wt\%, while $\mathrm{SiO}_{2}$ ranged from 1.69 - 31.21 wt\%. 
O. A. Ehinola et al.

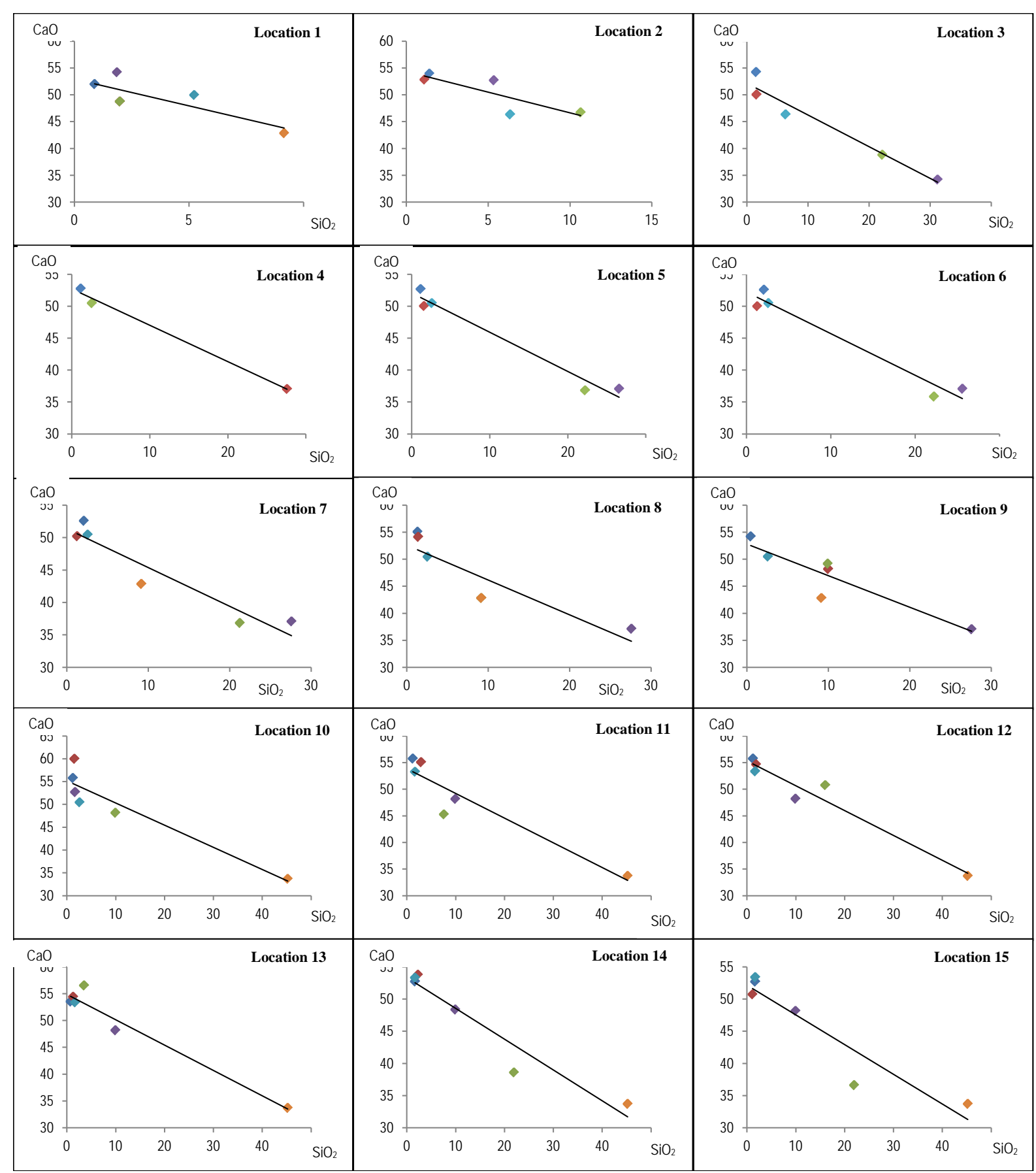

Figure 4. $\mathrm{SiO}_{2}$ (in wt\%) versus $\mathrm{CaO}$ (in wt\%) plots revealing a marked inverse relationship.

A 2-D and 3-D model views of $\mathrm{CaO}$ and $\mathrm{SiO}_{2}$ concentration is shown in Figure 7. Peaks of $\mathrm{CaO}$ and $\mathrm{SiO}_{2}$ concentrations were recorded in the southwestern and northwestern flanks respectively. Again, just as in bed $\mathrm{E}$ for a decline in $\mathrm{CaO}$ concentration there is a corresponding abundance of $\mathrm{SiO}_{2}$ was observed.

\subsection{3. $\mathrm{CaO}$ and $\mathrm{SiO}_{2}$ Variation in $\mathrm{Bed} \mathrm{C}$}

This bed is $3.5 \mathrm{~m}$ thick occupied depths of 8.6 - $9.3 \mathrm{~m}$ and 20.2 - $23.7 \mathrm{~m}$ at the upper and lower surfaces respectively. Table 1 showed $\mathrm{CaO}$ concentration of $35.84-56.52 \mathrm{wt} \%$, while $\mathrm{SiO}_{2}$ is $1.98-22.23 \mathrm{wt} \%$. Geochemical variation models of $\mathrm{CaO}$ and $\mathrm{SiO}_{2}$ indicated a decline in $\mathrm{CaO}$ (with small peaks in southern part) concentration 

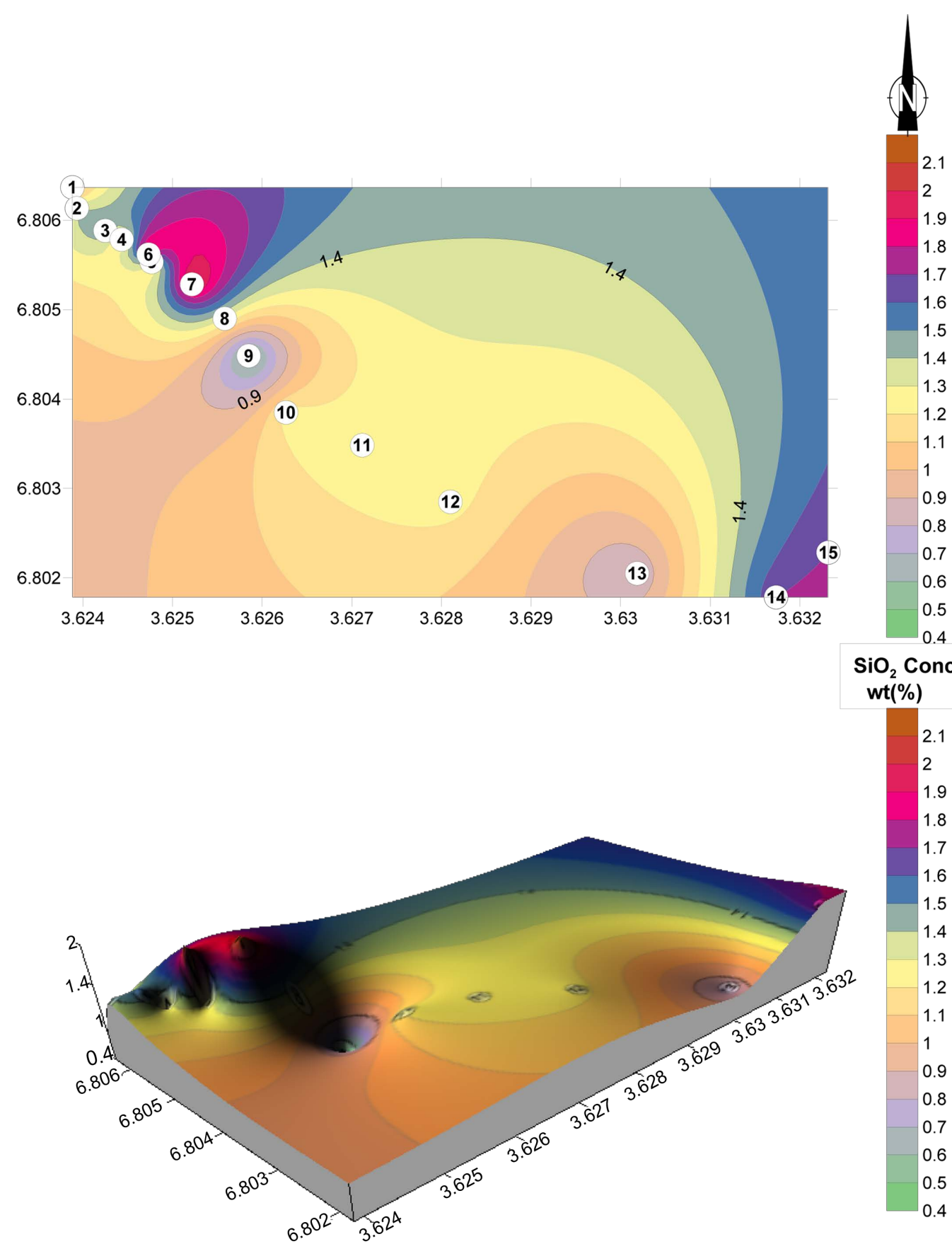

$\mathrm{SiO}_{2}$ Concentration wt(\%)

2.1

2

1.9

1.8

1.7

Figure 5. A generalized $\mathrm{SiO}_{2}$ (in wt\%) distribution models of the study area.

with an accompanying enrichment in $\mathrm{SiO}_{2}$ at both the southeastern and northwestern ends of the study area (Figure 8).

\subsection{4. $\mathrm{CaO}$ and $\mathrm{SiO}_{2}$ Variation in $\mathrm{Bed} \mathrm{B}$}

This 3 - $5 \mathrm{~m}$ thick bed occupied a depth of 7 - $25 \mathrm{~m}$ and 10 - $30 \mathrm{~m}$ at the upper and lower bedding planes respectively.

$\mathrm{SiO}_{2}$ content is 1.10 - $9.98 \mathrm{wt} \%$ and $\mathrm{CaO}$ is 48.19 - $59.99 \mathrm{wt} \%$ (Table 1). Figure 9 is the 2-D and 3-D model views of the bed $\mathrm{B}$, indicating a uniformly high $\mathrm{CaO}$ concentration with a sharp drop in the mid-western part. $\mathrm{SiO}_{2}$ concentration is very low in this bed; a peak concentration apparent in the mid-western part coincided with 


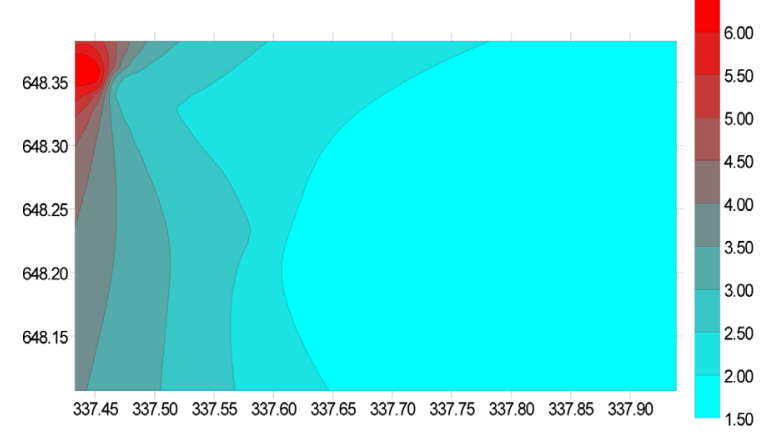

$\mathrm{SiO}_{2}$ concentration for bed $\mathrm{E}$ (map view).

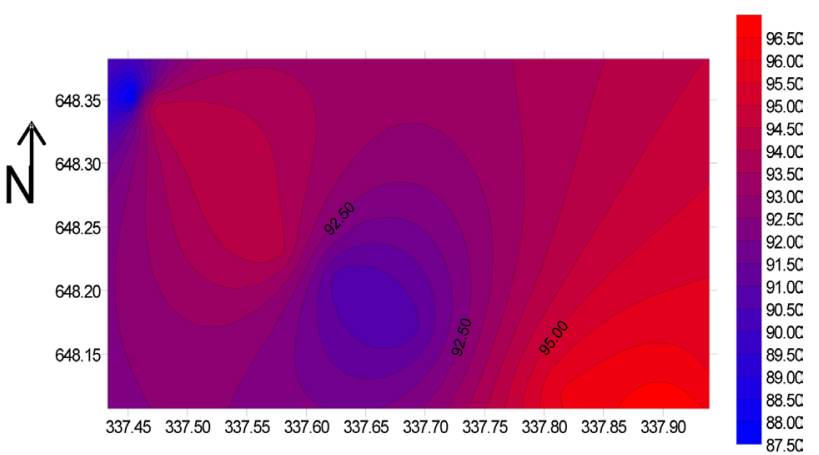

$\mathrm{CaO}$ concentration for bed $\mathrm{E}$ (map view).

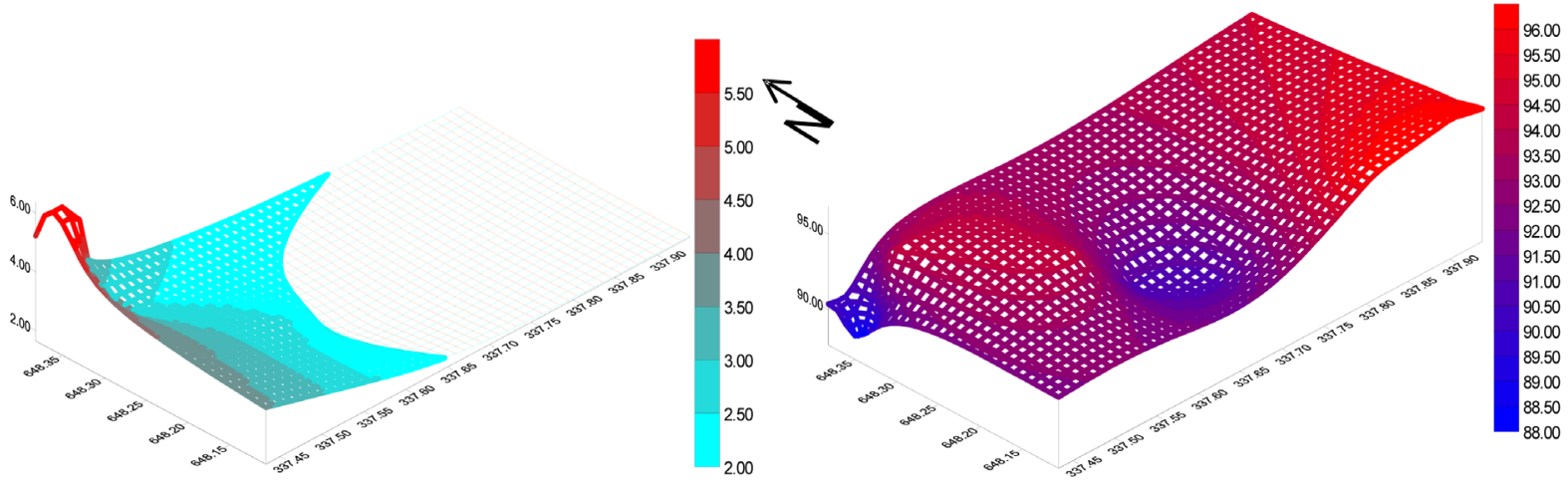

$\mathrm{SiO}_{2}$ concentration for bed $\mathrm{E}$ (3-D view).

Figure 6. Geochemical variation models of $\mathrm{SiO}_{2}$ (in wt\%) and $\mathrm{CaO}$ (in wt\%) for bed $\mathrm{E}$.

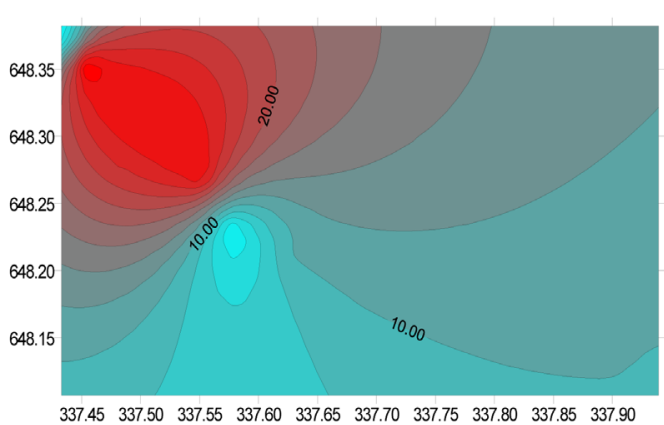

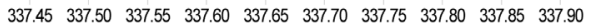

$\mathrm{SiO}_{2}$ concentration for bed $\mathrm{D}$ (map view).

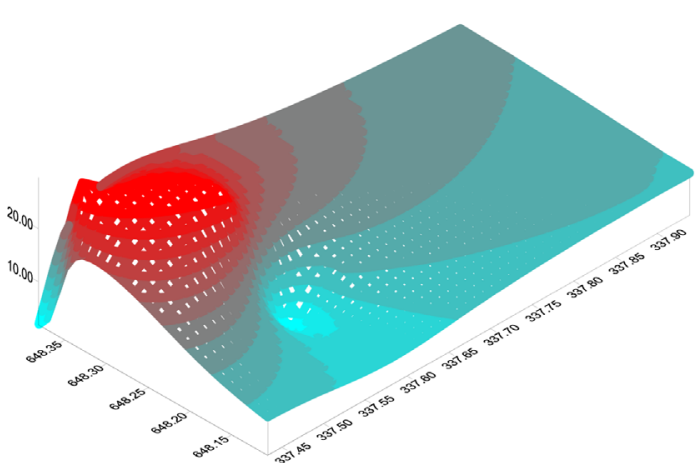

$\mathrm{SiO}_{2}$ concentration for bed $\mathrm{D}$ (3-D view).

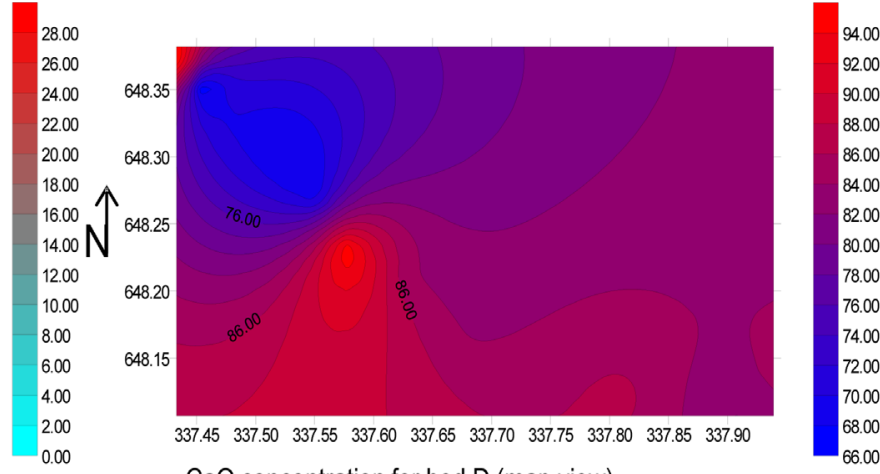

$\mathrm{CaO}$ concentration for bed $\mathrm{D}$ (map view).

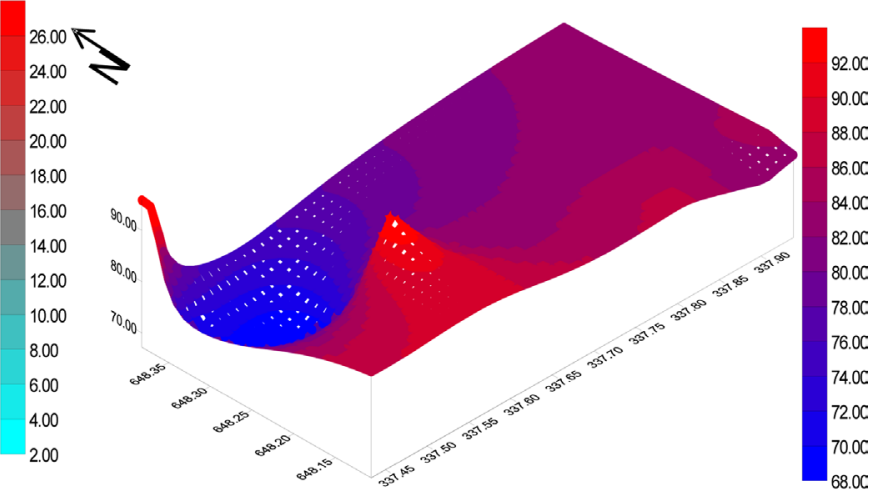

$\mathrm{CaO}$ concentration for bed $\mathrm{D}$ (3-D view)

Figure 7. Geochemical variation models of $\mathrm{SiO}_{2}$ (in wt \%) and $\mathrm{CaO}$ (in wt\%) for bed $\mathrm{D}$. 


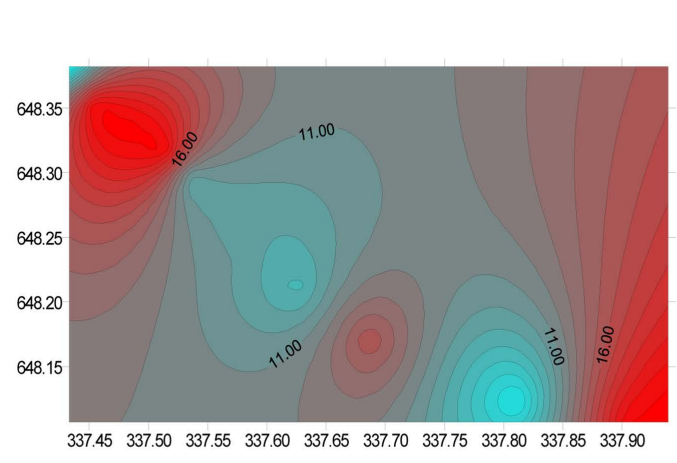

$\mathrm{SiO}_{2}$ concentration for bed $\mathrm{C}$ (map view).

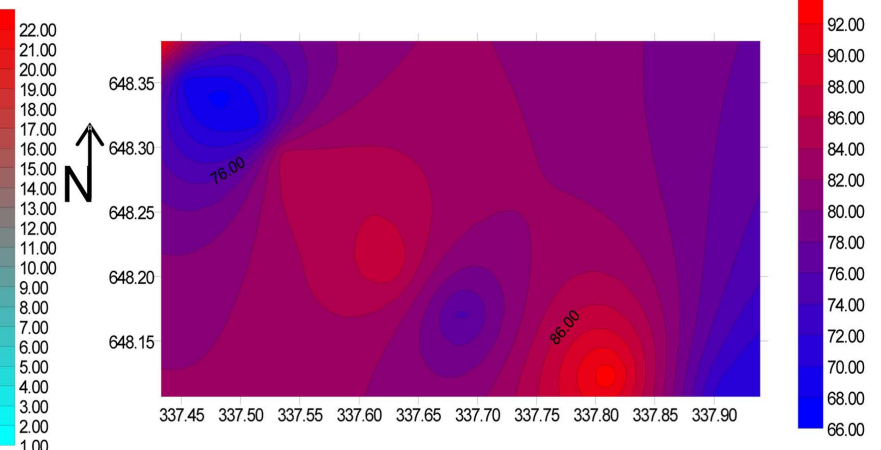

$\mathrm{CaO}$ concentration for bed $\mathrm{C}$ (map view).

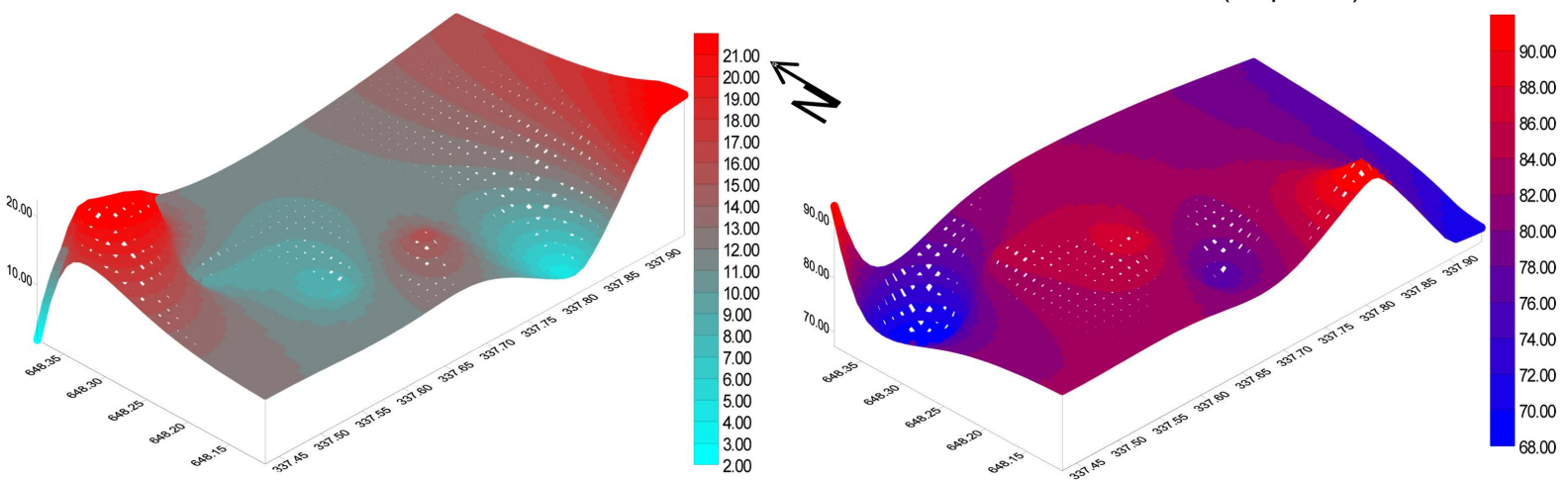

$\mathrm{SiO}_{2}$ concentration for bed $\mathrm{C}$ (3-D view).
$\mathrm{CaO}$ concentration for bed C (3-D view).

Figure 8. Geochemical variation models of $\mathrm{SiO}_{2}$ (in wt\%) and $\mathrm{CaO}$ (in wt\%) for bed $\mathrm{C}$.

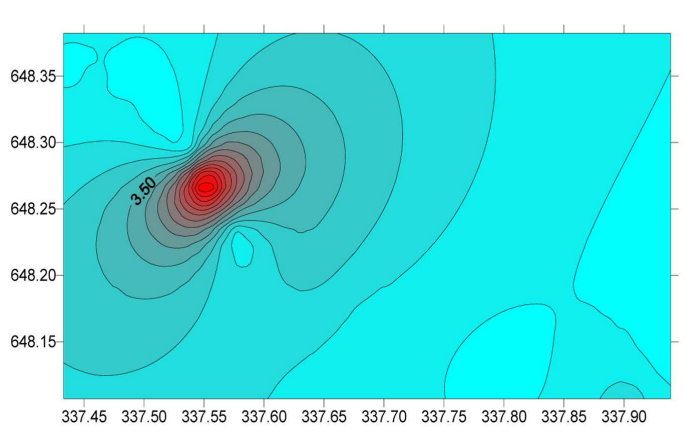

$\mathrm{SiO}_{2}$ concentration for bed $\mathrm{B}$ (map view).

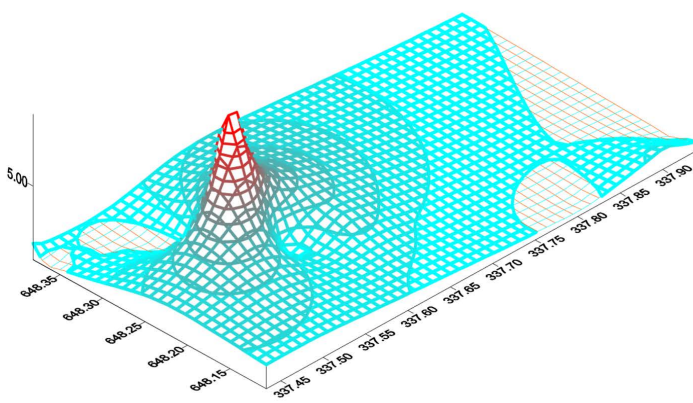

$\mathrm{SiO}_{2}$ concentration for bed $\mathrm{B}$ (3-D view).

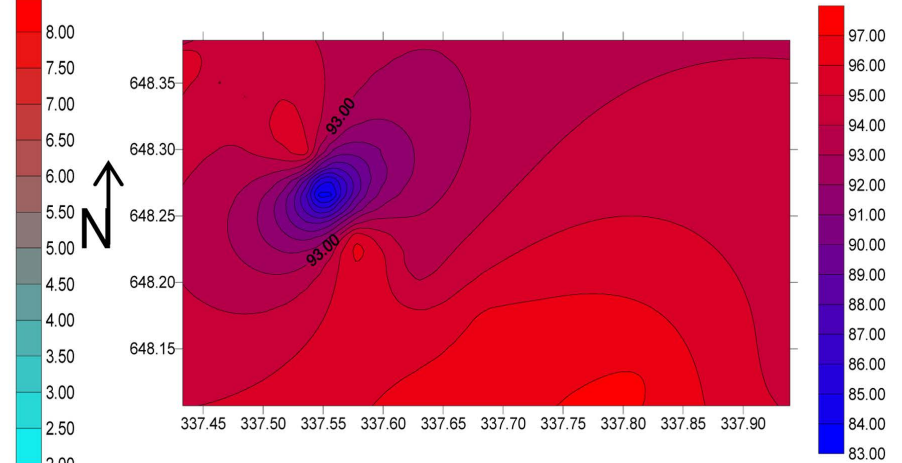

$\mathrm{CaO}$ concentration for bed $\mathrm{B}$ (map view).

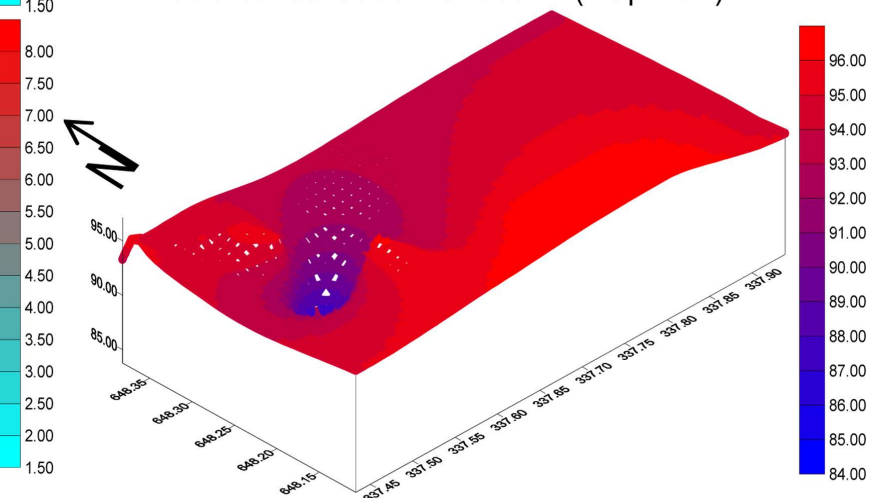

$\mathrm{CaO}$ concentration for bed $\mathrm{B}$ (3-D view).

Figure 9. Geochemical variation models of $\mathrm{SiO}_{2}$ (in wt\%) and $\mathrm{CaO}$ (in wt\%) for bed $\mathrm{B}$. 
the $\mathrm{CaO}$ lowest concentration. A notable increase in $\mathrm{CaO}$ concentration was noticed in compensation for the decline in $\mathrm{SiO}_{2}$ concentration, a trend similar to that of the aforementioned beds.

\subsection{5. $\mathrm{CaO}$ and $\mathrm{SiO}_{2}$ Variation in $\mathrm{Bed} \mathrm{A}$}

This bed is $\approx 5 \mathrm{~m}$ thick, occurring at depth range of 5 - $25 \mathrm{~m}$. For this bed, $\mathrm{CaO}$ content is $52.01-55.77 \mathrm{wt} \%$ while $\mathrm{SiO}_{2}$ is $0.47-2.07 \mathrm{wt} \%$ (Table 1 ). Figure 10 is the geochemical model for bed A in 2-D and 3-D views respectively. From this model, $\mathrm{CaO}$ indicated a peak concentration in the southwestern part, while $\mathrm{SiO}_{2}$ showed a high concentration running almost diagonally from the NNW part to the SSE part. This is shown by the corresponding "trough" on the model as against the "crests" of high $\mathrm{CaO}$ concentration.

\section{Discussion}

\subsection{Geochemistry, Provenance and Depositional Environment}

The silica content varies widely (0.47 - $3.21 \mathrm{wt} \%$, Table 1); an indication that the adjacent basement complex rocks of southwestern Nigeria may have sourced varied amounts of these detrital impurity and/or its dissolved component in the shallow marine environment. Geochemical plots (Figure 4) of $\mathrm{SiO}_{2}$ against $\mathrm{CaO}$ for all the samples analyzed clearly revealed a similar and unique trend marked by increase in $\mathrm{CaO}$ with corresponding decrease in $\mathrm{SiO}_{2}$ contents and vice versa. This $\mathrm{SiO}_{2}-\mathrm{CaO}$ negative correlation can be attributed to chemical diagenetic replacement. At the shallow part of the upper continental crust where silica is readily made available through weathering and erosion, calcite reacts with it to form a calc-silicate at low temperature and pressure. Also, at all stages of diagenesis, dissolved silica (derived from dissolution of siliceous tests of marine organisms) replaces calcite. Fluvial silica input often augment dissolved silica in the basin at the unset of shallow marine condition. However, $\mathrm{CaCO}_{3}$ production dominates as shallow marine conditions become fully established and silica supply from the hinterland reduces. Hence the higher the $\mathrm{SiO}_{2}$ input, the more the continental influence, whereas the $\mathrm{CaCO}_{3}$ production signals shallow marine incursion.

The low alumina content confirms a low index of weathering of the alumino-silicates such as feldspars and micas in the adjacent basement areas during transportation and deposition prior to diagenesis [18]. $\mathrm{Fe}_{2} \mathrm{O}_{3}$ is usually

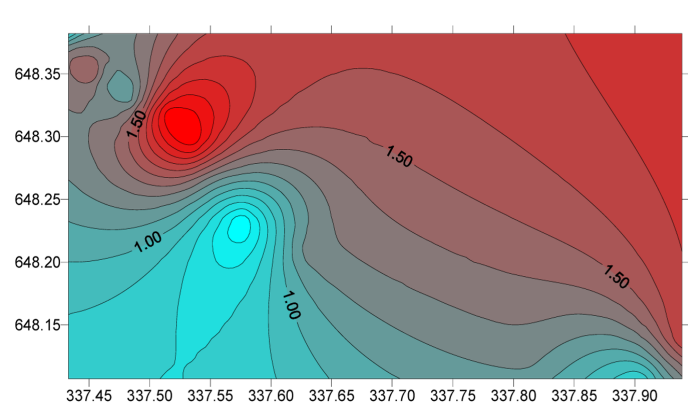

$\mathrm{SiO}_{2}$ concentration for bed $\mathrm{A}$ (map view).

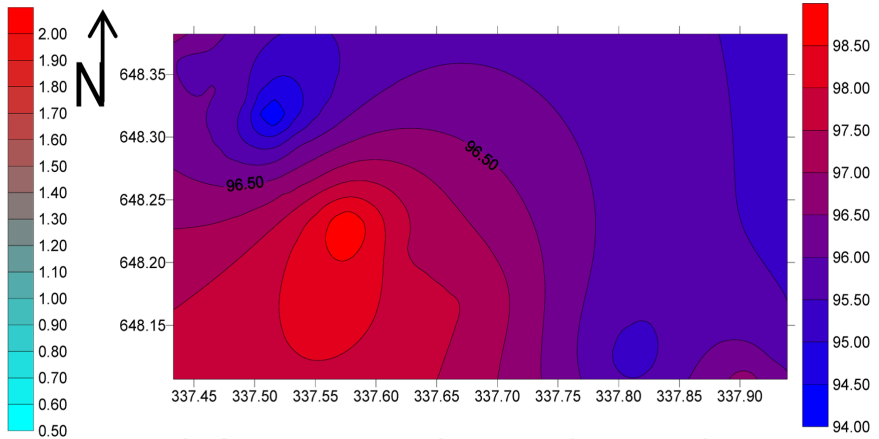

$\mathrm{CaO}$ concentration for bed $\mathrm{A}$ (map view).

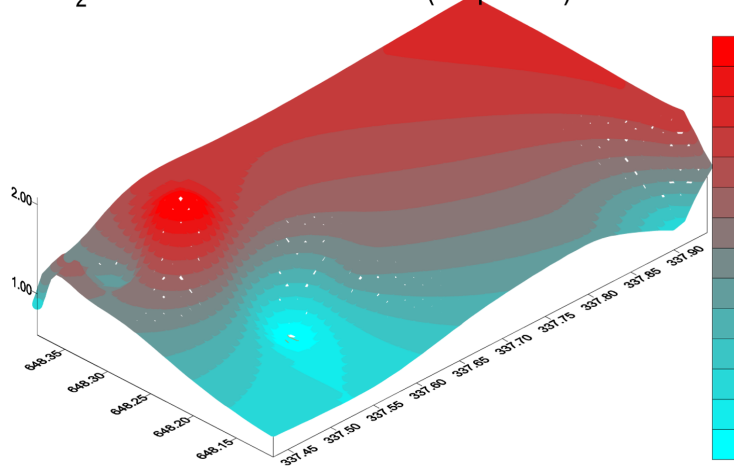

$\mathrm{SiO}_{2}$ concentration for bed $\mathrm{A}$ (3-D view).

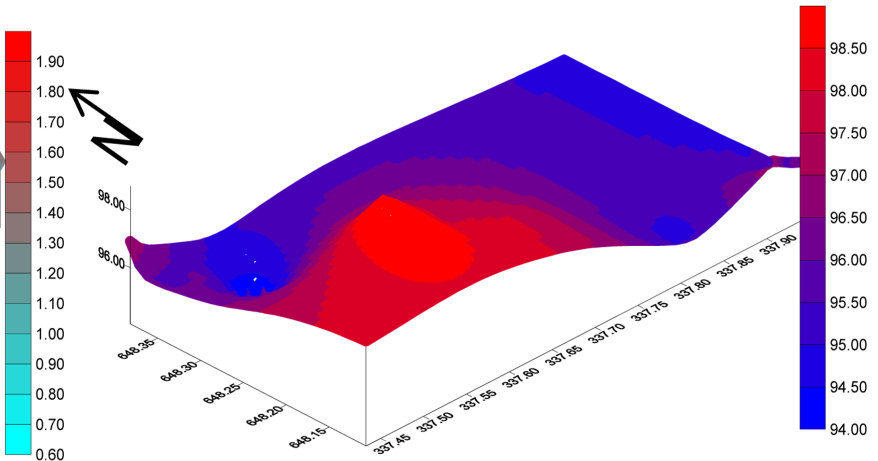

$\mathrm{CaO}$ concentration for bed $\mathrm{A}(3-\mathrm{D}$ view).

Figure 10. Geochemical variation models of $\mathrm{SiO}_{2}$ (in wt\%) and $\mathrm{CaO}$ (in wt\%) for bed $\mathrm{A}$. 
Table 2. Classification of calcium and magnesium contents of the Ewekoro limestone (After Todd [19]).

\begin{tabular}{|c|c|c|c|c|c|}
\hline Sample code & $\mathrm{CaO}(w \mathrm{t} \%)$ & MgO (wt\%) & Standard ratio $\mathrm{Ca} / \mathrm{Mg}$ & Reciprocal ratio $\mathrm{Mg} / \mathrm{Ca}$ & Descriptive term \\
\hline Bed A1 & 52.01 & 0.62 & 83.88 & 0.011 & Limestone \\
\hline Bed B1 & 48.78 & 0.83 & 58.77 & 0.017 & Limestone \\
\hline Bed C1 & 48.78 & 0.81 & 60.22 & 0.016 & Limestone \\
\hline Bed D1 & 54.22 & 0.64 & 84.71 & 0.011 & Limestone \\
\hline Bed E1 & 49.98 & 2.26 & 22.11 & 0.045 & Magnesian limestone \\
\hline Bed F1 & 42.84 & 1.4 & 30.60 & 0.032 & Magnesian limestone \\
\hline Bed A2 & 53.97 & 0.72 & 74.95 & 0.013 & Limestone \\
\hline Bed B2 & 52.85 & 0.78 & 67.75 & 0.014 & Limestone \\
\hline Bed C2 & 46.73 & 0.87 & 53.71 & 0.018 & Limestone \\
\hline Bed D2 & 52.7 & 0.64 & 82.34 & 0.012 & Limestone \\
\hline Bed E2 & 46.33 & 3.15 & 14.70 & 0.067 & Magnesian limestone \\
\hline Bed A3 & 54.24 & 1.24 & 43.74 & 0.022 & Limestone \\
\hline Bed B3 & 50.05 & 3.31 & 15.12 & 0.066 & Magnesian limestone \\
\hline Bed C3 & 38.81 & 3.14 & 12.35 & 0.080 & Dolomitic limestone \\
\hline Bed D3 & 34.22 & 0.62 & 55.19 & 0.018 & Limestone \\
\hline Bed E3 & 46.33 & 3.15 & 14.70 & 0.067 & Magnesian limestone \\
\hline Bed A4 & 52.75 & 1.72 & 30.66 & 0.032 & Magnesian limestone \\
\hline Bed D4 & 37.05 & 0.74 & 50.06 & 0.019 & Limestone \\
\hline Bed E4 & 50.48 & 0.75 & 67.30 & 0.014 & Limestone \\
\hline Bed A5 & 52.7 & 1.72 & 30.63 & 0.032 & Magnesian limestone \\
\hline Bed B5 & 50.04 & 3.31 & 15.11 & 0.066 & Magnesian limestone \\
\hline Bed C5 & 36.84 & 2.05 & 17.97 & 0.055 & Magnesian limestone \\
\hline Bed D5 & 37.06 & 0.74 & 50.08 & 0.019 & Limestone \\
\hline Bed E5 & 50.49 & 0.75 & 67.32 & 0.014 & Limestone \\
\hline Bed A6 & 52.58 & 1.03 & 51.04 & 0.019 & Limestone \\
\hline Bed B6 & 50.01 & 1.01 & 49.51 & 0.020 & Limestone \\
\hline Bed C6 & 35.84 & 2.05 & 17.48 & 0.057 & Magnesian limestone \\
\hline Bed D6 & 37.08 & 0.74 & 50.10 & 0.019 & Limestone \\
\hline Bed E6 & 50.48 & 0.75 & 67.30 & 0.014 & Limestone \\
\hline Bed A7 & 52.57 & 1.03 & 51.03 & 0.019 & Limestone \\
\hline Bed B7 & 50.2 & 1.01 & 49.70 & 0.020 & Limestone \\
\hline Bed C7 & 36.84 & 2.05 & 17.97 & 0.055 & Magnesian limestone \\
\hline Bed D7 & 37.09 & 0.74 & 50.12 & 0.019 & Limestone \\
\hline Bed E7 & 50.45 & 0.75 & 67.26 & 0.014 & Limestone \\
\hline Bed F7 & 42.84 & 1.4 & 30.60 & 0.032 & Magnesian limestone \\
\hline
\end{tabular}


O. A. Ehinola et al.

\section{Continued}

\begin{tabular}{|c|c|c|c|c|c|}
\hline Sample code & $\mathrm{CaO}(w \mathrm{t} \%)$ & MgO (wt \%) & Standard ratio $\mathrm{Ca} / \mathrm{Mg}$ & Reciprocal ratio $\mathrm{Mg} / \mathrm{Ca}$ & Descriptive term \\
\hline Bed A8 & 55.08 & 0.93 & 59.22 & 0.016 & Limestone \\
\hline Bed B8 & 54.13 & 0.61 & 88.73 & 0.011 & Limestone \\
\hline Bed C8 & 42.84 & 1.4 & 30.60 & 0.032 & Magnesian limestone \\
\hline Bed D8 & 37.12 & 0.74 & 50.16 & 0.019 & Limestone \\
\hline Bed E8 & 50.46 & 0.75 & 67.28 & 0.014 & Limestone \\
\hline Bed F8 & 42.84 & 1.4 & 30.60 & 0.032 & Magnesian limestone \\
\hline Bed A1 & 52.01 & 0.62 & 83.88 & 0.011 & Limestone \\
\hline Bed B1 & 48.78 & 0.83 & 58.77 & 0.017 & Limestone \\
\hline Bed C1 & 48.78 & 0.81 & 60.22 & 0.016 & Limestone \\
\hline Bed D1 & 54.22 & 0.64 & 84.71 & 0.011 & Limestone \\
\hline Bed E1 & 49.98 & 2.26 & 22.11 & 0.045 & Magnesian limestone \\
\hline Bed F1 & 42.84 & 1.4 & 30.60 & 0.032 & Magnesian limestone \\
\hline Bed A2 & 53.97 & 0.72 & 74.95 & 0.013 & Limestone \\
\hline Bed B2 & 52.85 & 0.78 & 67.75 & 0.014 & Limestone \\
\hline Bed C2 & 46.73 & 0.87 & 53.71 & 0.018 & Limestone \\
\hline Bed D2 & 52.7 & 0.64 & 82.34 & 0.012 & Limestone \\
\hline Bed E2 & 46.33 & 3.15 & 14.70 & 0.067 & Magnesian limestone \\
\hline Bed A3 & 54.24 & 1.24 & 43.74 & 0.022 & Limestone \\
\hline Bed B3 & 50.05 & 3.31 & 15.12 & 0.066 & Magnesian limestone \\
\hline Bed C3 & 38.81 & 3.14 & 12.35 & 0.080 & Dolomitic limestone \\
\hline Bed D3 & 34.22 & 0.62 & 55.19 & 0.018 & Limestone \\
\hline Bed E3 & 46.33 & 3.15 & 14.70 & 0.067 & Magnesian limestone \\
\hline Bed A4 & 52.75 & 1.72 & 30.66 & 0.032 & Magnesian limestone \\
\hline Bed D4 & 37.05 & 0.74 & 50.06 & 0.019 & Limestone \\
\hline Bed E4 & 50.48 & 0.75 & 67.30 & 0.014 & Limestone \\
\hline Bed A5 & 52.7 & 1.72 & 30.63 & 0.032 & Magnesian limestone \\
\hline Bed B5 & 50.04 & 3.31 & 15.11 & 0.066 & Magnesian limestone \\
\hline Bed C5 & 36.84 & 2.05 & 17.97 & 0.055 & Magnesian limestone \\
\hline Bed D5 & 37.06 & 0.74 & 50.08 & 0.019 & Limestone \\
\hline Bed E5 & 50.49 & 0.75 & 67.32 & 0.014 & Limestone \\
\hline Bed A6 & 52.58 & 1.03 & 51.04 & 0.019 & Limestone \\
\hline Bed B6 & 50.01 & 1.01 & 49.51 & 0.020 & Limestone \\
\hline Bed C6 & 35.84 & 2.05 & 17.48 & 0.057 & Magnesian limestone \\
\hline Bed D6 & 37.08 & 0.74 & 50.10 & 0.019 & Limestone \\
\hline Bed E6 & 50.48 & 0.75 & 67.30 & 0.014 & Limestone \\
\hline Bed A7 & 52.57 & 1.03 & 51.03 & 0.019 & Limestone \\
\hline
\end{tabular}




\begin{tabular}{cccccc} 
Continued & & & & & \\
\hline Bed B7 & 50.2 & 1.01 & 49.70 & 0.020 & Limestone \\
Bed C7 & 36.84 & 2.05 & 17.97 & 0.055 & Magnesian limestone \\
Bed D7 & 37.09 & 0.74 & 50.12 & 0.019 & Limestone \\
Bed E7 & 50.45 & 0.75 & 67.26 & 0.014 & Limestone \\
Bed F7 & 42.84 & 1.4 & 30.60 & 0.032 & Magnesian limestone \\
Bed A8 & 55.08 & 0.93 & 59.22 & 0.016 & Limestone \\
Bed B8 & 54.13 & 0.61 & 88.73 & 0.011 & Limestone \\
Bed C8 & 42.84 & 1.4 & 30.60 & 0.032 & Magnesian limestone \\
Bed D8 & 37.12 & 0.74 & 50.16 & 0.019 & Limestone \\
Bed E8 & 50.46 & 0.75 & 67.28 & 0.014 & Limestone \\
Bed F8 & 42.84 & 1.4 & 30.60 & 0.032 & Magnesian limestone \\
\hline
\end{tabular}

derived from intense chemical weathering of heavy mineral such as the ferromagnesians. Its low value indicates that the environment of deposition is a reducing one that does not favour the precipitation of Iron (II) to Iron (III) and thus leached away [18]. $\mathrm{SO}_{3}$ is low probably because anoxic conditions prevailed in such quiet, low energy environments and there is rapid rate of sulphate reduction.

\subsection{Implications of Ca/Mg and Mg/Ca Ratios for Palaeo-Salinity}

Todd [19] presented a petrogenetic classification of carbonate rocks that involved the standard ratio, Ca/Mg and reciprocal ratio, $\mathrm{Mg} / \mathrm{Ca}$. The class limits of the standard ratio, $\mathrm{Ca} / \mathrm{Mg}$ are: > 100 - 39.0, 39.0 - 12.3, 12.3 - 5.67, 5.67 - 1.86, 1.86 - 1.50, 1.50 - 1.22, and 1.22 - 1.00 expressed as limestone, magnesian limestone, dolomitic limestone, dolomitized limestone, calcareous dolomite, dolomite and magnesian dolomite respectively. Also, the class limits of the reciprocal ratio, $\mathrm{Mg} / \mathrm{Ca}$ are: 0 - 0.03, 0.03 - 0.08, 0.08 - 0.18, 0.18 - 0.54, 0.54 - 0.67, 0.67 0.82 and $0.82-1.00$ also expressed as limestone, magnesian limestone, dolomitic limestone, dolomitized limestone, calcareous dolomite, dolomite and magnesian dolomite respectively. The standard and reciprocal ratios of $\mathrm{Ca}$ and Mg composition of the Ewekoro limestone are shown in Table 2. More than $79 \%$ of the samples are classified as "pure" limestone and about $20 \%$ are magnesian limestone according to the method of [19]. The $\mathrm{Ca} / \mathrm{Mg}$ ratio has implications for the stability conditions of the depositional environment that led to the formation of the carbonate(s) [20]. Naturally, the $\mathrm{Mg} / \mathrm{Ca}$ ratio increases during evaporation of sea water, especially under saline environmental conditions. Considering the $\mathrm{Ca} / \mathrm{Mg}$ and $\mathrm{Mg} / \mathrm{Ca}$ ratios (Table 2) it can be concluded that the relative rate of evaporation of sea water and the palaeo-salinity condition was low, as such limestone was deposited more at the expense of dolomite. However, intermittent increase in rate of sea water evaporation and salinity resulted to the deposition of the few magnesian limestones.

\section{Conclusion}

The geochemical characterization of the limestones of the Ewekoro Formation through XRF analysis shed light on the level of chemical purity, provenance, diagenesis and environment of deposition of the study area. Geochemical variation model of $\mathrm{SiO}_{2}$ and $\mathrm{CaO}$ showed a general distribution of purity level of the limestone that is applicable to mining operations. The limestone is rich in $\mathrm{CaCO}_{3}$ with varied inputs of other oxides like $\mathrm{SiO}_{2}$, $\mathrm{MgO}, \mathrm{Fe}_{2} \mathrm{O}_{3}$, etc. A negative correlation resulted from $\mathrm{SiO}_{2}$ against $\mathrm{CaO}$ plots implying replacement chemical diagenesis under a shallow marine setting. Ratios of $\mathrm{Ca} / \mathrm{Mg}$ and $\mathrm{Mg} / \mathrm{Ca}$ revealed relatively low sea water evaporation and palaeo-salinity conditions that encouraged $\mathrm{CaCO}_{3}$ precipitation. However, occasional rise in sea water evaporation and salinity levels resulted in the formation of magnesian limestones.

\section{Acknowledgements}

The authors wish to acknowledge the Management of West African Portland Cement, Shagamu for permission to obtain samples and analyzing them. 


\section{References}

[1] Nwajide, C.S. (2013) Geology of Nigeria’s Sedimentary Basins. CSS Bookshop Ltd., Lagos.

[2] Jones, H.A. and Hockey, R.D. (1964) The Geology of Part of Southwestern Nigeria. Geological Survey of Nigeria Bulletin, 31, 1-101.

[3] Reyment, R.A. (1965) Aspects of the Geology of Nigeria. Ibadan University Press, Ibadan.

[4] Elueze, A.A. and Nton, M.E. (2004) Organic Geochemical Appraisal of Limestones and Shales in Part of Eastern Dahomey Basin, Southwestern Nigeria. Journal of Mining and Geology, 40, 29-40. http://dx.doi.org/10.4314/jmg.v40i1.18806

[5] Akinmonsin, A.A., Odewande and Akintola, A.I. (2005) Geochemical Composition and Textural Features of Some Carbonate Rocks in Parts of Southwestern Nigeria. Ife Journal of Science, 7, 101-111.

[6] Ajayi, T.R., Oyawale, A.A., Islander, F.Y., Asubiojo, O.I., Klein, D.E. and Adediran, A.I. (2006) Trace and Rare Earth Elements Geochemistry of Oshosun Sediments of Dahomey Basin, Southwestern Nigeria. Journal of Applied Science, 6, 2067-2076. http://dx.doi.org/10.3923/jas.2006.2067.2076

[7] Ofulume, A.B. (2012) Using Geochemical Criteria to Check the Depositional Environments Derived from Fossil Content and Microfacies of Shagamu, Mfamosing and Gboko Limestones, Nigeria. Journal of Applied Science Research, 8, 371-376.

[8] Ogbe, F.G.A. (1972) Stratigraphy of Strata Exposed in Ewekoro Quarry, Western Nigeria. In: Dessauvagie, T.F.J. and Whiteman, A.J., Eds., African Geology, University of Ibadan Press, Ibadan, 305-322.

[9] Omatsola, M.E. and Adegoke, O.S. (1981) Tectonic Evolution and Cretaceous Stratigraphy of the Dahomey Basin. Journal of Mining and Geology, 18, 130-137.

[10] Kingston, D.R., Dishroon, C.P. and Williams, P.A. (1983) Global Basin Classification System. American Association of Petroleum Geologist Bulletin, 67, 2175-2193.

[11] Burke, K., Dessauvagie, T.F.J. and Whiteman, A.J. (1971) The Opening of the Gulf of Guinea and Geological History of the Benue Depression and Niger Delta. Nature Physical Science, 233, 51-55. http://dx.doi.org/10.1038/physci233051a0

[12] Whiteman, A.J. (1982) Nigeria: Its Petroleum Geology, Resources and Potential. Graham and Trotman, London. http://dx.doi.org/10.1007/978-94-009-7361-9

[13] Billman, H.G. (1982) Offshore Stratigraphy and Paleontology of the Dahomey (Benin) Embayment, West Africa. Nigerian Association of Petroleum Explorationists Bulletin, 7, 121-130.

[14] Coker, S.L., Ejedawe, J.E. and Oshiorienua, J.A. (1983) Hydrocarbon Source Potential of Cretaceous Rock of Okitipupa Uplift. Nigerian Journal of Mining and Geology, 2, 163-169.

[15] Agagu, O.K. (1985) A Geological Guide to Bituminous Sediments in Southwestern Nigeria. Publication of Geology Department, University of Ibadan, Ibadan.

[16] Nton, M.E. (2001) Aspect of Rock Evaluation Studies of the Maastrichtian-Eocene Sediments. Journal of Mining and Geology, 13, 33-39.

[17] Fayose, E.A. and Azeez, L.O. (1972) Micropalaeontological Investigations of Ewekoro Area, Southwestern Nigeria. Micropaleontology, 18, 369-385. http://dx.doi.org/10.2307/1485014

[18] Brand, U. (1983) Mineralogy and Geochemistry of Deep Sea Clay in the Atlantic Ocean and Adjacent Seas and Ocean. Geological Society of America Bulletin, 76, 803-832.

[19] Todd, T.W. (1966) Petrogenetic Classification of Carbonate Rocks. Journal of Sedimentary Petrology, 36, 317-340.

[20] Marschner, H. (1968) Ca-Mg-Distribution in Carbonates from the Lower Keuper in NW-Germany. In: Muller, G. and Friedman, G.M., Eds., Recent Developments in Carbonate Sedimentology in Central Europe, Springer-Verlag, Berlin Heidelberg, 128-135. http://dx.doi.org/10.1007/978-3-642-88052-0_15 


\section{Submit or recommend next manuscript to SCIRP and we will provide best service for you:}

Accepting pre-submission inquiries through Email, Facebook, LinkedIn, Twitter, etc.

A wide selection of journals (inclusive of 9 subjects, more than 200 journals)

Providing 24-hour high-quality service

User-friendly online submission system

Fair and swift peer-review system

Efficient typesetting and proofreading procedure

Display of the result of downloads and visits, as well as the number of cited articles

Maximum dissemination of your research work

Submit your manuscript at: http://papersubmission.scirp.org/ 